\title{
Representations and cohomologies of Hom-Lie-Yamaguti algebras with applications
}

\author{
Tao Zhang ${ }^{a *}$ \\ ${ }^{a}$ College of Mathematics and Information Science, Henan Normal University, Xinxiang 453007, PR China
}

\begin{abstract}
The representation and cohomology theory of Hom-Lie-Yamaguti algebras is introduced. As an application, we study deformation and extension of Hom-Lie-Yamaguti algebras. It proved that a 1-parameter infinitesimal deformation of a Hom-Lie-Yamaguti algebra $T$ corresponds to a Hom-Lie-Yamaguti algebra of deformation type and a (2,3)-cocycle of $T$ with coefficients in the adjoint representation. We also prove that abelian extensions of Hom-LieYamaguti algebras are classified by the (2,3)-cohomology group.
\end{abstract}

\section{Introduction}

In recent years, Hom-type algebras were studied by many researchers. The first examples coming from $q$-deformations of Witt and Virasora algebras are Hom-Lie algebras, see 9. Other types include Hom-associative algebras, Hom-Nambu-Lie algebras, Hom-Hopf algebras, etc. See [1, 2, 3 , 5, 15, 18, 19, and the references therein.

In [8], the authors introduced the concept of Hom-Lie-Yamaguti algebras. It is a Hom-type generalization of a Lie-Yamaguti algebra in [11, 4, a general Lie triple system in [16, 17] and a Lie triple algebra in 10]. In 12, the authors studied the formal deformations of Hom-Lie-Yamaguti algebras, where only low dimensional deformation cohomology were defined without the help of any representation. So we wonder if there is a systematic study of Hom-Lie-Yamaguti algebras to give its general representation and cohomology theory? The present paper is denoted to solve this problem.

The method of this paper is based on our recent work [20, 21]. In [20], we give a new characterization of the representation and cohomology theory of Lie triple systems. In 21, we give a detailed study on the $(2,3)$-cohomology group associated to a representation of a Lie-Yamaguti algebra. As an application, we study deformation and extension theory of Lie-Yamaguti algebras. In this paper, we will first define the representation and cohomology theory of Hom-Lie-Yamaguti algebras. Then we will study deformation and extension theory of Hom-Lie-Yamaguti algebras as we did in 21. We will see that they are classified by the $(2,3)$-cohomology groups. The difficulty in this case is that, we have a morphism $\alpha: T \rightarrow T$ and more conditions with respect to $\alpha$ to be compatible with the structure of Hom-Lie-Yamaguti algebras. Fortunately, we overcome this difficulty by using an equivalent characterization of representation and a careful analysis on the coboundary operator. All our results in this paper will generalize Yamaguti's representation and cohomology theory for Lie-Yamaguti algebras in [16, 17.

The paper is organized as follows. In section 2, we introduce the concept of representations of a Hom-Lie-Yamaguti algebra. Then we define the coboundary operator on the cochain complex of a Hom-Lie-Yamaguti algebra with coefficient in a representation $V$ to produce the cohomology

\footnotetext{
*Corresponding author. E-mail address: zhangtao@htu.cn

${ }^{0}$ Keyword: Hom-Lie-Yamaguti algebra, representation, cohomology, deformation, abelian extensions

${ }^{0}$ Mathematics Subject Classification (2010): 17A40, 17A30, 17B56
} 
group. We pay special attentions to the $(2,3)$-cohomology group since it will used in the following context. In Section 3, we study the infinitesimal deformation theory of Hom-Lie-Yamaguti algebras. We prove that there is a Hom-Lie-Yamaguti algebra of deformation type and a $(2,3)$-cocycle of $T$ with coefficients in the adjoint representation associated to a deformation. We also introduce the notion of Nijenhuis operators to describe trivial deformations. In Section 4, we study abelian extensions of Hom-Lie-Yamaguti algebras. We prove that there is a one-to-one correspondence between equivalence classes of abelian extensions of the Hom-Lie-Yamaguti algebra $T$ by $V$ and elements of the $(2,3)$-cohomology group.

Throughout this paper, we work on an algebraically closed field $\mathbb{K}$ of characteristic different from 2 and 3.

\section{Representations and Cohomologies}

In this section, we first recall some basic definitions regarding Hom-Lie-Yamaguti algebras. Then we define the representation and cohomology theory of Hom-Lie-Yamaguti algebras.

A Hom-vector space is a pair $(V, \alpha)$ in which $V$ is a vector space and $\alpha: V \rightarrow V$ is a linear map. A morphism $(V, \alpha) \rightarrow(W, \beta)$ of Hom-vector space is a linear map $f: V \rightarrow W$ such that $\beta \circ f=f \circ \alpha$. We will often abbreviate a Hom-vector space $(V, \alpha)$ to $V$.

Definition 2.1. A Hom-Lie-Yamaguti algebra (or HLYA for short) consists of a vector space $T$ together with a linear map $\alpha: T \rightarrow T$, a bilinear map $[\cdot, \cdot]: T \times T \rightarrow T$ and a trilinear map $[\cdot, \cdot, \cdot]: T \times T \times T \rightarrow T$ such that, for all $x_{i}, y_{i} \in T$, the following conditions are satisfied:

- $\left(\operatorname{HLY01)} \alpha\left(\left[x_{1}, x_{2}\right]\right)=\left[\alpha\left(x_{1}\right), \alpha\left(x_{2}\right)\right]\right.$;

- $(\mathrm{HLY02}) \quad \alpha\left(\left[x_{1}, x_{2}, x_{3}\right]\right)=\left[\alpha\left(x_{1}\right), \alpha\left(x_{2}\right), \alpha\left(x_{3}\right)\right]$;

- $(\mathrm{HLY} 1) \quad\left[x_{1}, x_{2}\right]+\left[x_{2}, x_{1}\right]=0$;

- $\left(\right.$ HLY2) $\left[x_{1}, x_{2}, x_{3}\right]+\left[x_{2}, x_{1}, x_{3}\right]=0$;

- $\left(\right.$ HLY3) $\left[\left[x_{1}, x_{2}\right], \alpha\left(x_{3}\right)\right]+$ c.p. $+\left[x_{1}, x_{2}, x_{3}\right]+$ c.p. $=0$;

- (HLY4) $\left[\left[x_{1}, x_{2}\right], \alpha\left(x_{3}\right), \alpha\left(y_{1}\right)\right]+\left[\left[x_{2}, x_{3}\right], \alpha\left(x_{1}\right), \alpha\left(y_{1}\right)\right]+\left[\left[x_{3}, x_{1}\right], \alpha\left(x_{2}\right), \alpha\left(y_{1}\right)\right]=0$;

- $(\mathrm{HLY} 5) \quad\left[\alpha\left(x_{1}\right), \alpha\left(x_{2}\right),\left[y_{1}, y_{2}\right]\right]=\left[\left[x_{1}, x_{2}, y_{1}\right], \alpha^{2}\left(y_{2}\right)\right]+\left[\alpha^{2}\left(y_{1}\right),\left[x_{1}, x_{2}, y_{2}\right]\right]$

- $\left(\right.$ HLY6) $\left[\alpha^{2}\left(x_{1}\right), \alpha^{2}\left(x_{2}\right),\left[y_{1}, y_{2}, y_{3}\right]\right]=\left[\left[x_{1}, x_{2}, y_{1}\right], \alpha^{2}\left(y_{2}\right), \alpha^{2}\left(y_{3}\right)\right]+\left[\alpha^{2}\left(y_{1}\right),\left[x_{1}, x_{2}, y_{2}\right], \alpha^{2}\left(y_{3}\right)\right]+$ $\left[\alpha^{2}\left(y_{1}\right), \alpha^{2}\left(y_{2}\right),\left[x_{1}, x_{2}, y_{3}\right]\right]$.

where c.p. means cyclic permutations with respect to $x_{1}, x_{2}, x_{3}$. We denote a HLYA by $(T,[\cdot, \cdot],[\cdot, \cdot, \cdot], \alpha)$ or simply by $T$.

A linear map $\alpha$ satisfying (HLY01) and (HLY02) is called an algebraic homomorphism. When $\alpha=\mathrm{id}$, conditions (HLY01) and (HLY02) are trivial and the other conditions (HLY1)-(HLY6) reduced to conditions (LY1)-(LY6) for a Lie-Yamaguti algebra (LYA for short) in [21. Note that conditions (HLY1) and (HLY2) are equivalent to $\left[x_{1}, x_{1}\right]=0$ and $\left[x_{1}, x_{1}, x_{3}\right]=0$ respectively.

A homomorphism between two HLYAs $T$ and $T^{\prime}$ is a linear map $\varphi: T \rightarrow T^{\prime}$ satisfying $\varphi \circ \alpha=$ $\alpha^{\prime} \circ \varphi$ and

$$
\varphi\left(\left[x_{1}, x_{2}\right]\right)=\left[\varphi\left(x_{1}\right), \varphi\left(x_{2}\right)\right]^{\prime}, \quad \varphi\left(\left[x_{1}, x_{2}, x_{3}\right]\right)=\left[\varphi\left(x_{1}\right), \varphi\left(x_{2}\right), \varphi\left(x_{3}\right)\right]^{\prime} .
$$

Definition 2.2. A HLYA of deformation type consists of a vector space $T$ together with a linear map $\alpha: T \rightarrow T$, a bilinear map $\nu: T \times T \rightarrow T$ and a trilinear map $\omega: T \times T \times T \rightarrow T$ satisfying all conditions in Definition 2.1 except that (HLY3) is replaced by the following conditions: 
- (HLY3') $\nu\left(\nu\left(x_{1}, x_{2}\right), \alpha\left(x_{3}\right)\right)+c . p .=0$.

Now we give the definition of a representation of a HLYA.

Definition 2.3. Let $(T, \alpha)$ be a HLYA and $(V, \beta)$ be a Hom-vector space. A representation of $(T, \alpha)$ on $(V, \beta)$ consists of a linear map $\rho: T \rightarrow \operatorname{End}(V)$ and bilinear maps $D, \theta: T \times T \rightarrow \operatorname{End}(V)$ such that the following conditions are satisfied:

- $(\operatorname{HR} 01) \quad \rho\left(\alpha\left(x_{1}\right)\right) \circ \beta=\beta \circ \rho\left(x_{1}\right)$;

- $(\operatorname{HR} 02) \quad D\left(\alpha\left(x_{1}\right), \alpha\left(x_{2}\right)\right) \circ \beta=\beta \circ D\left(x_{1}, x_{2}\right)$;

- (HR03) $\theta\left(\alpha\left(x_{1}\right), \alpha\left(x_{2}\right)\right) \circ \beta=\beta \circ \theta\left(x_{1}, x_{2}\right)$;

- $(\operatorname{HR} 31) \quad D\left(x_{1}, x_{2}\right)-\theta\left(x_{2}, x_{1}\right)+\theta\left(x_{1}, x_{2}\right)+\rho\left(\left[x_{1}, x_{2}\right]\right) \circ \beta-\rho\left(\alpha\left(x_{1}\right)\right) \rho\left(x_{2}\right)+\rho\left(\alpha\left(x_{2}\right)\right) \rho\left(x_{1}\right)=0$;

- (HR41) $D\left(\left[x_{1}, x_{2}\right], \alpha\left(x_{3}\right)\right)+D\left(\left[x_{2}, x_{3}\right], \alpha\left(x_{1}\right)\right)+D\left(\left[x_{3}, x_{1}\right], \alpha\left(x_{2}\right)\right)=0$

- $(\operatorname{HR} 42) \quad \theta\left(\left[x_{1}, x_{2}\right], \alpha\left(y_{1}\right)\right) \circ \beta=\theta\left(\alpha\left(x_{1}\right), \alpha\left(y_{1}\right)\right) \rho\left(x_{2}\right)-\theta\left(\alpha\left(x_{2}\right), \alpha\left(y_{1}\right)\right) \rho\left(x_{1}\right)$;

- (HR51) $D\left(\alpha\left(x_{1}\right), \alpha\left(x_{2}\right)\right) \rho\left(y_{2}\right)=\rho\left(\alpha^{2}\left(y_{2}\right)\right) D\left(x_{1}, x_{2}\right)+\rho\left(\left[x_{1}, x_{2}, y_{2}\right]\right) \circ \beta^{2} . ;$

- $(\operatorname{HR} 52) \quad \theta\left(\alpha\left(x_{1}\right),\left[y_{1}, y_{2}\right]\right) \circ \beta=\rho\left(\alpha^{2}\left(y_{1}\right)\right) \theta\left(x_{1}, y_{2}\right)-\rho\left(\alpha^{2}\left(y_{2}\right)\right) \theta\left(x_{1}, y_{1}\right)$;

- $(\operatorname{HR} 61) \quad D\left(\alpha^{2}\left(x_{1}\right), \alpha^{2}\left(x_{2}\right)\right) \theta\left(y_{1}, y_{2}\right)$

$$
=\theta\left(\alpha^{2}\left(y_{1}\right), \alpha^{2}\left(y_{2}\right)\right) D\left(x_{1}, x_{2}\right)+\theta\left(\left[x_{1}, x_{2}, y_{1}\right], \alpha^{2}\left(y_{2}\right)\right) \circ \beta^{2}+\theta\left(\alpha^{2}\left(y_{1}\right),\left[x_{1}, x_{2}, y_{2}\right]\right) \circ \beta^{2} ;
$$

- $(\operatorname{HR} 62) \quad \theta\left(\alpha^{2}\left(x_{1}\right),\left[y_{1}, y_{2}, y_{3}\right]\right) \circ \beta^{2}$

$$
=\theta\left(\alpha^{2}\left(y_{2}\right), \alpha^{2}\left(y_{3}\right)\right) \theta\left(x_{1}, y_{1}\right)-\theta\left(\alpha^{2}\left(y_{1}\right), \alpha^{2}\left(y_{3}\right)\right) \theta\left(x_{1}, y_{2}\right)+D\left(\alpha^{2}\left(y_{1}\right), \alpha^{2}\left(y_{2}\right)\right) \theta\left(x_{1}, y_{3}\right) .
$$

In this case, we also call $V$ to be a $T$-module.

For example, given a HLYA $T$, there is a natural adjoint representation on itself. The corresponding representation maps $\rho, D$ and $\theta$ are given by

$$
\rho\left(x_{1}\right)\left(x_{2}\right):=\left[x_{1}, x_{2}\right], \quad D\left(x_{1}, x_{2}\right) x_{3}:=\left[x_{1}, x_{2}, x_{3}\right], \quad \theta\left(x_{1}, x_{2}\right) x_{3}:=\left[x_{3}, x_{1}, x_{2}\right] .
$$

The next Proposition 2.4 gives an equivalent characterization of a representation. The proof is omitted since it is same as the proof of Lemma 4.5 in the last section.

Proposition 2.4. Let $(T, \alpha)$ be a HLYA and $(V, \beta)$ be a Hom-vector space. Assume we have a map $\rho$ from $T$ to $\operatorname{End}(V)$ and maps $D, \theta$ from $T \times T$ to $\operatorname{End}(V)$. Then $(\rho, D, \theta)$ is a representation of $T$ on $V$ if and only if $T \oplus V$ is a HLYA under the following maps:

$$
\begin{aligned}
(\alpha+\beta)\left(x_{1}+u_{1}\right) \triangleq & \alpha\left(x_{1}\right)+\beta\left(u_{1}\right), \\
{\left[x_{1}+u_{1}, x_{2}+u_{2}\right] \triangleq } & {\left[x_{1}, x_{2}\right]+\rho\left(x_{1}\right)\left(u_{2}\right)-\rho\left(x_{2}\right)\left(u_{1}\right), } \\
{\left[x_{1}+u_{1}, x_{2}+u_{2}, x_{3}+u_{3}\right] \triangleq } & {\left[x_{1}, x_{2}, x_{3}\right]+D\left(x_{1}, x_{2}\right)\left(u_{3}\right)-\theta\left(x_{1}, x_{3}\right)\left(u_{2}\right) } \\
& +\theta\left(x_{2}, x_{3}\right)\left(u_{1}\right),
\end{aligned}
$$

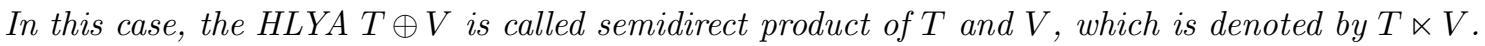

Motivated by Yamaguti's cohomology for Lie-Yamaguti algebras, we are going to define cohomology for HLYAs as follows. 
Let $V$ be a representation of HLYA $T$. Let us define the cohomology groups of $T$ with coefficients in $V$. Let $f: T \times \cdots \times T \rightarrow V$ be $n$-linear maps of $T$ into $V$ such that the following conditions are satisfied:

$$
\begin{aligned}
f\left(\alpha\left(x_{1}\right) \cdots, \alpha\left(x_{n}\right)\right) & =\beta\left(f\left(x_{1}, \cdots, x_{n}\right)\right), \\
f\left(x_{1}, \cdots, x_{2 i-1}, x_{2 i} \cdots, x_{n}\right) & =0, \text { if } x_{2 i-1}=x_{2 i} .
\end{aligned}
$$

The vector space spanned by such linear maps is called an $n$-cochain of $T$, which is denoted by $C^{n}(T, V)$ for $n \geq 1$.

Definition 2.5. For any $(f, g) \in C^{2 n}(T, V) \times C^{2 n+1}(T, V)$ the coboundary operator $\delta:(f, g) \mapsto$ $\left(\delta_{\mathrm{I}} f, \delta_{\mathrm{II}} g\right)$ is a mapping from $C^{2 n}(T, V) \times C^{2 n+1}(T, V)$ into $C^{2 n+2}(T, V) \times C^{2 n+3}(T, V)$ defined as follows:

$$
\begin{aligned}
& \left(\delta_{\mathrm{I}} f\right)\left(x_{1}, x_{2}, \cdots, x_{2 n+2}\right) \\
= & \left.\rho\left(\alpha^{2 n}\left(x_{2 n+1}\right)\right) g\left(x_{1}, \cdots, x_{2 n}, x_{2 n+2}\right)\right)-\rho\left(\alpha^{2 n}\left(x_{2 n+2}\right)\right) g\left(x_{1}, \cdots, x_{2 n+1}\right) \\
& -g\left(\alpha\left(x_{1}\right), \cdots, \alpha\left(x_{2 n}\right),\left[x_{2 n+1}, x_{2 n+2}\right]\right) \\
& +\sum_{k=1}^{n}(-1)^{n+k+1} D\left(\alpha^{2 n-1}\left(x_{2 k-1}\right), \alpha^{2 n-1}\left(x_{2 k}\right)\right) f\left(x_{1}, \cdots, \hat{x}_{2 k-1}, \hat{x}_{2 k}, \cdots, x_{2 n+2}\right) \\
& +\sum_{k=1}^{n} \sum_{j=2 k+1}^{2 n+2}(-1)^{n+k} f\left(\alpha^{2}\left(x_{1}\right), \cdots, \hat{x}_{2 k-1}, \hat{x}_{2 k}, \cdots,\left[x_{2 k-1}, x_{2 k}, x_{j}\right], \cdots, \alpha^{2}\left(x_{2 n+2}\right)\right), \\
= & \left(\delta_{\mathrm{II}} g\right)\left(x_{1}, x_{2}, \cdots, x_{2 n+3}\right) \\
& \theta\left(\alpha^{2 n}\left(x_{2 n+2}\right), \alpha^{2 n}\left(x_{2 n+3}\right)\right) g\left(x_{1}, \cdots, x_{2 n+1}\right) \\
& -\theta\left(\alpha^{2 n}\left(x_{2 n+1}\right), \alpha^{2 n}\left(x_{2 n+3}\right)\right) g\left(x_{1}, \cdots, x_{2 n}, x_{2 n+2}\right) \\
& +\sum_{k=1}^{n+1}(-1)^{n+k+1} D\left(\alpha^{2 n}\left(x_{2 k-1}\right), \alpha^{2 n}\left(x_{2 k}\right)\right) g\left(x_{1}, \cdots, \hat{x}_{2 k-1}, \hat{x}_{2 k}, \cdots, x_{2 n+3}\right) \\
& +\sum_{k=1}^{n+1} \sum_{j=2 k+1}^{2 n+3}(-1)^{n+k} g\left(\alpha^{2}\left(x_{1}\right), \cdots, \hat{x}_{2 k-1}, \hat{x}_{2 k}, \cdots,\left[x_{2 k-1}, x_{2 k}, x_{j}\right], \cdots, \alpha^{2}\left(x_{2 n+3}\right)\right) .
\end{aligned}
$$

When $\alpha=$ id, one recovers Yamaguti's cohomology for LYA in [17.

Lemma 2.6. With the above notations, for any $(f, g) \in C^{2 n}(T, V) \times C^{2 n+1}(T, V)$, we have

$$
\begin{aligned}
\delta_{I} f\left(\alpha\left(x_{1}\right) \cdots, \alpha\left(x_{2 n+2}\right)\right) & =\beta\left(\delta_{I} f\left(x_{1}, x_{2}, \cdots, x_{2 n+2}\right)\right), \\
\delta_{I I} g\left(\alpha\left(x_{1}\right) \cdots, \alpha\left(x_{2 n+3}\right)\right) & =\beta\left(\delta_{I I} g\left(x_{1}, x_{2}, \cdots, x_{2 n+3}\right)\right) .
\end{aligned}
$$

Thus we obtain a well-defined map

$$
\delta=\left(\delta_{I}, \delta_{I I}\right): C^{2 n}(T, V) \times C^{2 n+1}(T, V) \rightarrow C^{2 n+2}(T, V) \times C^{2 n+3}(T, V) .
$$

Proof. We only prove equation (7) since equation (8) can be verified similarly. By Definition 2.5, we have

$$
\begin{aligned}
& \left(\delta_{\mathrm{I}} f\right)\left(\alpha\left(x_{1}\right), \cdots, \alpha\left(x_{2 n+2}\right)\right) \\
= & \rho\left(\alpha^{2 n+1}\left(x_{2 n+1}\right)\right) g\left(\alpha\left(x_{1}\right), \cdots, \alpha\left(x_{2 n+2}\right)\right)-\rho\left(\alpha^{2 n+1}\left(x_{2 n+2}\right)\right) g\left(\alpha\left(x_{1}\right), \cdots, \alpha\left(x_{2 n+1}\right)\right) \\
& -g\left(\alpha^{2}\left(x_{1}\right), \cdots, \alpha^{2}\left(x_{2 n}\right), \alpha\left(\left[x_{2 n+1}, x_{2 n+2}\right]\right)\right) \\
& +\sum_{k=1}^{n}(-1)^{n+k+1} D\left(\alpha^{2 n}\left(x_{2 k-1}\right), \alpha^{2 n}\left(x_{2 k}\right)\right) f\left(\alpha\left(x_{1}\right), \cdots, \hat{x}_{2 k-1}, \hat{x}_{2 k}, \cdots, \alpha\left(x_{2 n+2}\right)\right)
\end{aligned}
$$




$$
\begin{aligned}
& +\sum_{k=1}^{n} \sum_{j=2 k+1}^{2 n+2}(-1)^{n+k} f\left(\alpha^{3}\left(x_{1}\right), \cdots, \hat{x}_{2 k-1}, \hat{x}_{2 k}, \cdots, \alpha\left(\left[x_{2 k-1}, x_{2 k}, x_{j}\right]\right), \cdots, \alpha^{3}\left(x_{2 n+2}\right)\right) \\
= & \rho\left(\alpha^{2 n+1}\left(x_{2 n+1}\right)\right) \circ \beta\left(g\left(x_{1}, \cdots, x_{2 n+2}\right)\right)-\rho\left(\alpha^{2 n+1}\left(x_{2 n+2}\right)\right) \circ \beta\left(g\left(x_{1}, \cdots, x_{2 n+1}\right)\right) \\
& -\beta\left(g\left(\alpha\left(x_{1}\right), \cdots, \alpha\left(x_{2 n}\right),\left[x_{2 n+1}, x_{2 n+2}\right]\right)\right) \\
& +\sum_{k=1}^{n}(-1)^{n+k+1} D\left(\alpha^{2 n}\left(x_{2 k-1}\right), \alpha^{2 n}\left(x_{2 k}\right)\right) \circ \beta\left(f\left(x_{1}, \cdots, \hat{x}_{2 k-1}, \hat{x}_{2 k}, \cdots, x_{2 n+2}\right)\right) \\
& +\sum_{k=1}^{n} \sum_{j=2 k+1}^{2 n+2}(-1)^{n+k} \beta\left(f\left(\alpha^{2}\left(x_{1}\right), \cdots, \hat{x}_{2 k-1}, \hat{x}_{2 k}, \cdots,\left[x_{2 k-1}, x_{2 k}, x_{j}\right], \cdots, \alpha^{2}\left(x_{2 n+2}\right)\right)\right) \\
= & \left.\beta\left(\rho\left(\alpha^{2 n}\left(x_{2 n+1}\right)\right) g\left(x_{1}, \cdots, x_{2 n}, x_{2 n+2}\right)\right)\right)-\beta\left(\rho\left(\alpha^{2 n}\left(x_{2 n+2}\right)\right) g\left(x_{1}, \cdots, x_{2 n+1}\right)\right) \\
& -\beta\left(g\left(\alpha\left(x_{1}\right), \cdots, \alpha\left(x_{2 n}\right),\left[x_{2 n+1}, x_{2 n+2}\right]\right)\right) \\
& +\sum_{k=1}^{n}(-1)^{n+k+1} \beta\left(D\left(\alpha^{2 n-1}\left(x_{2 k-1}\right), \alpha^{2 n-1}\left(x_{2 k}\right)\right) f\left(x_{1}, \cdots, \hat{x}_{2 k-1}, \hat{x}_{2 k}, \cdots, x_{2 n+2}\right)\right) \\
& +\sum_{k=1}^{n} \sum_{j=2 k+1}^{2 n+2}(-1)^{n+k} \beta\left(f\left(\alpha^{2}\left(x_{1}\right), \cdots, \hat{x}_{2 k-1}, \hat{x}_{2 k}, \cdots,\left[x_{2 k-1}, x_{2 k}, x_{j}\right], \cdots, \alpha^{2}\left(x_{2 n+2}\right)\right)\right) \\
= & \beta\left(\left(\delta_{\mathrm{I}} f\right)\left(x_{1}, x_{2}, \cdots, x_{2 n+2}\right)\right)
\end{aligned}
$$

where in the second equality we use condition (5) and in the third equality we use conditions (HR01)-(HR03).

Proposition 2.7. The coboundary operator defined above satisfies $\delta \circ \delta=0$, that is $\delta_{I} \circ \delta_{I}=0$ and $\delta_{I I} \circ \delta_{I I}=0$.

The above Proposition 2.7 can be proved by tedious computations. For our purpose, we will check a special case in Proposition 2.11.

Let $Z^{2 n}(T, V) \times Z^{2 n+1}(T, V)$ be the subspace of $C^{2 n}(T, V) \times C^{2 n+1}(T, V)$ spanned by $(f, g)$ such that $\delta(f, g)=0$ which is called the space of cocycles and $B^{2 n}(T, V) \times B^{2 n+1}(T, V)=$ $\delta\left(C^{2 n-2}(T, V) \times C^{2 n-1}(T, V)\right)$ which is called the space of coboundaries.

Definition 2.8. For the case $n \geq 2$, the $(2 n, 2 n+1)$-cohomology group of a HLYA $T$ with coefficients in $V$ is defined to be the quotient space:

$$
H^{2 n}(T, V) \times H^{2 n+1}(T, V) \triangleq\left(Z^{2 n}(T, V) \times Z^{2 n+1}(T, V)\right) /\left(B^{2 n}(T, V) \times B^{2 n+1}(T, V)\right) .
$$

In conclusion, we obtain a cochain complex whose cohomology group is called cohomology group of a HLYA $T$ with coefficients in $V$.

Note that in the above Definition 2.12 we assume $n \geq 2$. For the case $n=1$, we define the $(2,3)$-cohomology group of a HLYA $T$ with coefficients in $V$ as follows.

Let $C^{2}(T, V)$ be the space of maps $\nu: T \times T \rightarrow V$ such that $\nu\left(x_{1}, x_{2}\right)=-\nu\left(x_{2}, x_{1}\right)$ and

- $(\mathrm{CC} 01) \quad \nu\left(\alpha\left(x_{1}\right), \alpha\left(x_{2}\right)\right)=\beta \circ \nu\left(x_{1}, x_{2}\right)$.

Let $C^{3}(T, V)$ be the space of maps $\omega: T \times T \times T \rightarrow V$ such that $\omega\left(x_{1}, x_{2}, x_{3}\right)=-\omega\left(x_{2}, x_{1}, x_{3}\right)$ and

- $(\mathrm{CC} 02) \omega\left(\alpha\left(x_{1}\right), \alpha\left(x_{2}\right), \alpha\left(x_{3}\right)\right)=\beta \circ \omega\left(x_{1}, x_{2}, x_{3}\right)$.

Definition 2.9. Let $(T, \alpha)$ be a HLYA and $(V, \beta)$ a $T$-module. Then $(\nu, \omega) \in C^{2}(T, V) \times C^{3}(T, V)$ is called a $(2,3)$-cocycle if for all $x_{1}, x_{2}, y_{1}, y_{2}, y_{3} \in T$, we have 
- (CC1) $\omega\left(x_{1}, x_{2}, x_{3}\right)+$ c.p. $-\rho\left(\alpha\left(x_{1}\right)\right) \nu\left(x_{2}, x_{3}\right)-c . p .+\nu\left(\left[x_{1}, x_{2}\right], \alpha\left(x_{3}\right)\right)+c . p .=0$

- (CC2) $\theta\left(\alpha\left(x_{1}\right), \alpha\left(y_{1}\right)\right) \nu\left(x_{2}, x_{3}\right)+c . p .+\omega\left(\left[x_{1}, x_{2}\right], \alpha\left(x_{3}\right), \alpha\left(y_{1}\right)\right)+c . p .=0$;

- $(\mathrm{CC} 3)$

$$
\begin{aligned}
& \omega\left(\alpha\left(x_{1}\right), \alpha\left(x_{2}\right),\left[y_{1}, y_{2}\right]\right)+D\left(\alpha\left(x_{1}\right), \alpha\left(x_{2}\right)\right) \omega\left(y_{1}, y_{2}\right) \\
= & \left.\nu\left(\left[x_{1}, x_{2}, y_{1}\right], \alpha^{2}\left(y_{2}\right)\right)+\nu\left(\alpha^{2}\left(y_{1}\right),\left[x_{1}, x_{2}, y_{2}\right]\right)\right) \\
& +\rho\left(\alpha^{2}\left(y_{1}\right)\right) \omega\left(x_{1}, x_{2}, y_{2}\right)-\rho\left(\alpha^{2}\left(y_{2}\right)\right) \omega\left(x_{1}, x_{2}, y_{1}\right) ;
\end{aligned}
$$

- $(\mathrm{CC} 4)$

$$
\begin{aligned}
& \omega\left(\alpha^{2}\left(x_{1}\right), \alpha^{2}\left(x_{2}\right),\left[y_{1}, y_{2}, y_{3}\right]\right)+D\left(\alpha^{2}\left(x_{1}\right), \alpha^{2}\left(x_{2}\right)\right) \omega\left(y_{1}, y_{2}, y_{3}\right) \\
= & \omega\left(\left[x_{1}, x_{2}, y_{1}\right], \alpha^{2}\left(y_{2}\right), \alpha^{2}\left(y_{3}\right)\right)+\omega\left(\alpha^{2}\left(y_{1}\right),\left[x_{1}, x_{2}, y_{2}\right], \alpha^{2}\left(y_{3}\right)\right) \\
& +\omega\left(\alpha^{2}\left(y_{1}\right), \alpha^{2}\left(y_{2}\right),\left[x_{1}, x_{2}, y_{3}\right]\right)+\theta\left(\alpha^{2}\left(y_{2}\right), \alpha^{2}\left(y_{3}\right)\right) \omega\left(x_{1}, x_{2}, y_{1}\right) \\
& -\theta\left(\alpha^{2}\left(y_{1}\right), \alpha^{2}\left(y_{3}\right)\right) \omega\left(x_{1}, x_{2}, y_{2}\right)+D\left(\alpha^{2}\left(y_{1}\right), \alpha^{2}\left(y_{2}\right)\right) \omega\left(x_{1}, x_{2}, y_{3}\right) .
\end{aligned}
$$

The space of $(2,3)$-cocycles is denoted by $Z^{2}(T, V) \times Z^{3}(T, V)$.

We remark that the conditions $(\mathrm{CC} 3)$ and $(\mathrm{CC} 4)$ are equivalent to $\delta_{\mathrm{I}}(\nu)=0$ and $\delta_{\mathrm{II}}(\omega)=0$ respectively. Why we add conditions $(\mathrm{CC} 1)$ and $(\mathrm{CC} 2)$ can be seen from the following context.

Let $f$ be a linear mapping of $T$ into a representation space $V$. Then $f$ is called a derivation of $T$ into $V$ if

$$
\begin{aligned}
f\left(\left[x_{1}, x_{2}\right]\right) & =\rho\left(x_{1}\right) f\left(x_{2}\right)-\rho\left(x_{2}\right) f\left(x_{1}\right), \\
f\left(\left[x_{1}, x_{2}, x_{3}\right]\right) & =\theta\left(x_{2}, x_{3}\right) f\left(x_{1}\right)-\theta\left(x_{1}, x_{3}\right) f\left(x_{2}\right)+D\left(x_{1}, x_{2}\right) f\left(x_{3}\right) .
\end{aligned}
$$

Definition 2.10. Let $(T, \alpha)$ be a HLYA and $(V, \beta)$ a $T$-module. Then $(\nu, \omega) \in C^{2}(T, V) \times C^{3}(T, V)$ is called a (2,3)-coboundary if there exists a map $f: T \rightarrow V$ such that

- (BB01) $f \circ \alpha=\beta \circ f$;

- (BB1) $\nu\left(x_{1}, x_{2}\right)=\rho\left(x_{1}\right) f\left(x_{2}\right)-\rho\left(x_{2}\right) f\left(x_{1}\right)-f\left(\left[x_{1}, x_{2}\right]\right)$;

- (BB2) $\omega\left(x_{1}, x_{2}, x_{3}\right)=\theta\left(x_{2}, x_{3}\right) f\left(x_{1}\right)-\theta\left(x_{1}, x_{3}\right) f\left(x_{2}\right)+D\left(x_{1}, x_{2}\right) f\left(x_{3}\right)-f\left(\left[x_{1}, x_{2}, x_{3}\right]\right)$.

The space of $(2,3)$-coboundaries is denoted by $B^{2}(T, V) \times B^{3}(T, V)$.

Proposition 2.11. The space of (2,3)-coboundaries is contained in space of (2,3)-cocycles.

Proof. We will verify that if $(\nu, \omega)$ satisfies (BB01), (BB1) and (BB2), then it must satisfies conditions $(\mathrm{CC} 01),(\mathrm{CC} 02)$ and $(\mathrm{CC} 1)-(\mathrm{CC} 4)$.

By definition, for (CC01), we have

$$
\begin{aligned}
& \nu\left(\alpha\left(x_{1}\right), \alpha\left(x_{2}\right)\right)-\beta \circ \nu\left(x_{1}, x_{2}\right) \\
& =\rho\left(\alpha\left(x_{1}\right)\right) f\left(\alpha\left(x_{2}\right)\right)-\rho\left(\alpha\left(x_{2}\right)\right) f\left(\alpha\left(x_{1}\right)\right)-f\left(\left[\alpha\left(x_{1}\right), \alpha\left(x_{2}\right)\right]\right) \\
& -\beta \circ\left\{\rho\left(x_{1}\right) f\left(x_{2}\right)-\rho\left(x_{2}\right) f\left(x_{1}\right)-f\left(\left[x_{1}, x_{2}\right]\right)\right\} \\
& =\underline{\rho\left(\alpha\left(x_{1}\right)\right) \circ \beta} \circ f\left(x_{2}\right)-\underline{\rho\left(\alpha\left(x_{2}\right)\right) \circ \beta} \circ f\left(x_{1}\right)-f \circ \alpha\left(\left[x_{1}, x_{2}\right]\right) \\
& -\underline{\beta \circ \rho\left(x_{1}\right)} \circ f\left(x_{2}\right)+\underline{\beta \circ \rho\left(x_{2}\right)} \circ f\left(x_{1}\right)+\beta \circ f\left(\left[x_{1}, x_{2}\right]\right) \\
& =0 \text {. }
\end{aligned}
$$

where in the last equality we have used (HR01) and (BB01). 
For (CC02), we have

$$
\begin{aligned}
& \omega\left(\alpha\left(x_{1}\right), \alpha\left(x_{2}\right), \alpha\left(x_{3}\right)\right)-\beta \circ \omega\left(x_{1}, x_{2}, x_{3}\right) \\
& =\theta\left(\alpha\left(x_{2}\right), \alpha\left(x_{3}\right)\right) f\left(\alpha\left(x_{1}\right)\right)-\theta\left(\alpha\left(x_{1}\right), \alpha\left(x_{3}\right)\right) f\left(\alpha\left(x_{2}\right)\right) \\
& +D\left(\alpha\left(x_{1}\right), \alpha\left(x_{2}\right)\right) f\left(\alpha\left(x_{3}\right)\right)-f\left(\left[\alpha\left(x_{1}\right), \alpha\left(x_{2}\right), \alpha\left(x_{3}\right)\right]\right) \\
& \beta \circ\left\{\theta\left(x_{2}, x_{3}\right) f\left(x_{1}\right)-\theta\left(x_{1}, x_{3}\right) f\left(x_{2}\right)+D\left(x_{1}, x_{2}\right) f\left(x_{3}\right)-f\left(\left[x_{1}, x_{2}, x_{3}\right]\right)\right\} \\
& =\underline{\theta\left(\alpha\left(x_{2}\right), \alpha\left(x_{3}\right)\right) \circ \beta} \circ f\left(x_{1}\right)-\underline{\underline{\theta\left(\alpha\left(x_{1}\right), \alpha\left(x_{3}\right)\right) \circ \beta}} \circ f\left(x_{2}\right) \\
& +D\left(\alpha\left(x_{1}\right), \alpha\left(x_{2}\right)\right) \circ \beta \circ f\left(x_{3}\right)-f \circ \alpha\left(\left[x_{1}, x_{2}, x_{3}\right]\right) \\
& \underline{\overline{\beta \circ \theta\left(x_{2}, x_{3}\right)} \circ f\left(x_{1}\right)}-\underline{\underline{\beta \circ \theta\left(x_{1}, x_{3}\right)}} \circ f\left(x_{2}\right)+\underline{\underline{\underline{\beta \circ D\left(x_{1}, x_{2}\right)}}} \circ f\left(x_{3}\right) \\
& -\beta \circ f\left(\left[x_{1}, x_{2}, x_{3}\right]\right) \\
& =0 \text {. }
\end{aligned}
$$

where in the last equality we have used (HR02), (HR03) and (BB01).

For (CC1), we have

$$
\begin{aligned}
& \omega\left(x_{1}, x_{2}, x_{3}\right)+c . p .-\rho\left(\alpha\left(x_{1}\right)\right) \nu\left(x_{2}, x_{3}\right)-c . p .+\nu\left(\left[x_{1}, x_{2}\right], \alpha\left(x_{3}\right)\right)+c . p . \\
= & \left(\theta\left(x_{2}, x_{3}\right) f\left(x_{1}\right)-\theta\left(x_{1}, x_{3}\right) f\left(x_{2}\right)+D\left(x_{1}, x_{2}\right) f\left(x_{3}\right)-f\left(\left[x_{1}, x_{2}, x_{3}\right]\right)\right)+c . p . \\
& -\rho\left(\alpha\left(x_{1}\right)\right)\left(\rho\left(x_{2}\right) f\left(x_{3}\right)-\rho\left(x_{3}\right) f\left(x_{2}\right)-f\left(\left[x_{2}, x_{3}\right]\right)\right)+c . p . \\
& +\left(\rho\left(\left[x_{1}, x_{2}\right]\right) f\left(\alpha\left(x_{3}\right)\right)-\rho\left(\alpha\left(x_{3}\right)\right) f\left(\left[x_{1}, x_{2}\right]\right)-f\left(\left[\left[x_{1}, x_{2}\right], \alpha\left(x_{3}\right)\right]\right)\right)+c . p . \\
=\quad & \left(D\left(x_{1}, x_{2}\right)-\theta\left(x_{2}, x_{1}\right)+\theta\left(x_{1}, x_{2}\right)+\rho\left[x_{1}, x_{2}\right] \circ \beta\right. \\
& \left.-\rho\left(\alpha\left(x_{1}\right)\right) \rho\left(x_{2}\right)+\rho\left(\alpha\left(x_{2}\right)\right) \rho\left(x_{1}\right)\right) f\left(x_{3}\right)+c \cdot p . \\
& -f\left(\left[x_{1}, x_{2}, x_{3}\right]+c \cdot p .+\left[\left[x_{1}, x_{2}\right], \alpha\left(x_{3}\right)\right]+c . p .\right) \\
= & 0 .
\end{aligned}
$$

The last equality is by (HR31) and (HLY3).

By direct computations, for (CC2), we get

$$
\begin{aligned}
& \theta\left(\alpha\left(x_{1}\right), \alpha\left(y_{1}\right)\right) \nu\left(x_{2}, x_{3}\right)+c . p .+\omega\left(\left[x_{1}, x_{2}\right], \alpha\left(x_{3}\right), \alpha\left(y_{1}\right)\right)+c . p . \\
=\quad & \theta\left(\alpha\left(x_{1}\right), \alpha\left(y_{1}\right)\right)\left(\rho\left(x_{2}\right) f\left(x_{3}\right)-\rho\left(x_{3}\right) f\left(x_{2}\right)-f\left(\left[x_{2}, x_{3}\right]\right)\right)+c . p . \\
& +\left(\theta\left(\alpha\left(x_{3}\right), \alpha\left(y_{1}\right)\right) f\left(\left[x_{1}, x_{2}\right]\right)-\theta\left(\left[x_{1}, x_{2}\right], \alpha\left(y_{1}\right)\right) f\left(\alpha\left(x_{3}\right)\right)\right. \\
& \left.+D\left(\left[x_{1}, x_{2}\right], \alpha\left(x_{3}\right)\right) f\left(\alpha\left(y_{1}\right)\right)-f\left(\left[\left[x_{1}, x_{2}\right], \alpha\left(x_{3}\right), \alpha\left(y_{1}\right)\right]\right)\right)+c . p . \\
=\quad & \left(D\left(\left[x_{1}, x_{2}\right], \alpha\left(x_{3}\right)\right)+D\left(\left[x_{2}, x_{3}\right], \alpha\left(x_{1}\right)\right)+D\left(\left[x_{3}, x_{1}\right], \alpha\left(x_{2}\right)\right)\right) f\left(\alpha\left(y_{1}\right)\right) \\
& -\left(\theta\left(\left[x_{1}, x_{2}\right], y_{1}\right) \circ \beta-\theta\left(\alpha\left(x_{1}\right), \alpha\left(y_{1}\right)\right) \rho\left(x_{2}\right)+\theta\left(\alpha\left(x_{2}\right), \alpha\left(y_{1}\right)\right) \rho\left(x_{1}\right)\right) f\left(x_{3}\right)-c . p . \\
& -f\left(\left[\left[x_{1}, x_{2}\right], \alpha\left(x_{3}\right), \alpha\left(y_{1}\right)\right]+c \cdot p .\right) \\
= & 0 .
\end{aligned}
$$

The last equality is by (HR41), (HR42) and (HLY4).

The other cases can checked as follows: (CC3) is valid by conditions (HR51), (HR52) and (HLY5); (CC4) is valid by conditions (HR61), (HR62) and (HLY6). Therefore the space of $(2,3)$ coboundaries is contained in space of $(2,3)$-cocycles. The proof is finished. 
Definition 2.12. The (2,3)-cohomology group of a HLYA $T$ with coefficients in $V$ is defined as the quotient space

$$
H^{2}(T, V) \times H^{3}(T, V) \triangleq Z^{2}(T, V) \times Z^{3}(T, V) / B^{2}(T, V) \times B^{3}(T, V) .
$$

\section{Infinitesimal Deformations}

Let $T$ be a HLYA and $\nu: T \times T \rightarrow T$ and $\omega: T \times T \times T \rightarrow T$ be bilinear and trilinear maps. Consider a $\lambda$-parametrized family of bilinear maps and trilinear maps:

$$
\begin{aligned}
{\left[x_{1}, x_{2}\right]_{\lambda} } & \triangleq\left[x_{1}, x_{2}\right]+\lambda \nu\left(x_{1}, x_{2}\right), \\
{\left[x_{1}, x_{2}, x_{3}\right]_{\lambda} } & \triangleq\left[x_{1}, x_{2}, x_{3}\right]+\lambda \omega\left(x_{1}, x_{2}, x_{3}\right) .
\end{aligned}
$$

If $[\cdot, \cdot]_{\lambda}$ and $[\cdot, \cdot, \cdot]_{\lambda}$ endow $T$ with a HLYA structure which is denoted by $T_{\lambda}$, then we say that $(\nu, \omega)$ generates a $\lambda$-parameter infinitesimal deformation of HLYA $T$.

Theorem 3.1. With the above notations, $(\nu, \omega)$ generates a $\lambda$-parameter infinitesimal deformation of a HLYA T if and only if the following two conditions hold:

(i) $(\nu, \omega)$ defines a HLYA of deformation type on $T$;

(ii) $(\nu, \omega)$ is a (2,3)-cocycle of $T$ with coefficients in the adjoint representation.

Proof. Assume $(\nu, \omega)$ generates a $\lambda$-parameter infinitesimal deformation of the HLYA $T$, then the maps $\left[x_{1}, x_{2}\right]_{\lambda}$ and $\left[x_{1}, x_{2}, x_{3}\right]_{\lambda}$ defined above must satisfies conditions (HLY1)-(HLY6). From these conditions, we will deduce that $(\nu, \omega)$ is a $(2,3)$-cocycle and $(\nu, \omega)$ defines a HLYA of deformation type on $T$.

From (HLY01), we have

$$
\begin{aligned}
& \alpha\left(\left[x_{1}, x_{2}\right]_{\lambda}\right)-\left[\alpha\left(x_{1}\right), \alpha\left(x_{2}\right)\right]_{\lambda} \\
= & \alpha\left[x_{1}, x_{2}\right]-\left[\alpha\left(x_{1}\right), \alpha\left(x_{2}\right)\right]+\lambda\left\{\alpha \circ \nu\left(x_{1}, x_{2}\right)-\nu\left(\alpha\left(x_{1}\right), \alpha\left(x_{2}\right)\right)\right\} \\
= & 0 .
\end{aligned}
$$

thus we get

$$
\alpha \circ \nu\left(x_{1}, x_{2}\right)=\nu\left(\alpha\left(x_{1}\right), \alpha\left(x_{2}\right)\right) .
$$

From (HLY02), we have

$$
\begin{aligned}
& \alpha\left(\left[x_{1}, x_{2}, x_{3}\right]_{\lambda}\right)-\left[\alpha\left(x_{1}\right), \alpha\left(x_{2}\right), \alpha\left(x_{3}\right)\right]_{\lambda} \\
= & \alpha\left[x_{1}, x_{2}, x_{3}\right]-\left[\alpha\left(x_{1}\right), \alpha\left(x_{2}\right), \alpha\left(x_{3}\right)\right] \\
& +\lambda\left\{\alpha \circ \nu\left(x_{1}, x_{2}, x_{3}\right)-\nu\left(\alpha\left(x_{1}\right), \alpha\left(x_{2}\right), \alpha\left(x_{3}\right)\right)\right\} \\
= & 0
\end{aligned}
$$

thus we obtain

$$
\alpha \circ \nu\left(x_{1}, x_{2}, x_{3}\right)=\nu\left(\alpha\left(x_{1}\right), \alpha\left(x_{2}\right), \alpha\left(x_{3}\right)\right) .
$$

From (HLY3), we have

$$
\begin{aligned}
& {\left[x_{1}, x_{2}, x_{3}\right]_{\lambda}+c . p .+\left[\left[x_{1}, x_{2}\right]_{\lambda}, \alpha\left(x_{3}\right)\right]_{\lambda}+c . p . } \\
= & {\left[x_{1}, x_{2}, x_{3}\right]+c . p .+\left[\left[x_{1}, x_{2}\right], \alpha\left(x_{3}\right)\right]+c . p . } \\
& +\lambda\left\{\omega\left(x_{1}, x_{2}, x_{3}\right)+c . p .+\nu\left(\left[x_{1}, x_{2}\right], \alpha\left(x_{3}\right)\right)+c . p .+\left[\nu\left(x_{1}, x_{2}\right), \alpha\left(x_{3}\right)\right]+c . p .\right\} \\
& +\lambda^{2}\left\{\nu\left(\nu\left(x_{1}, x_{2}\right), \alpha\left(x_{3}\right)\right)+c . p .\right\}
\end{aligned}
$$


thus we get

$$
\begin{array}{r}
\omega\left(x_{1}, x_{2}, x_{3}\right)+c . p .+\nu\left(\left[x_{1}, x_{2}\right], \alpha\left(x_{3}\right)\right)+c . p .+\left[\nu\left(x_{1}, x_{2}\right), \alpha\left(x_{3}\right)\right]+c . p .=0 \\
\nu\left(\nu\left(x_{1}, x_{2}\right), \alpha\left(x_{3}\right)\right)+c . p .=0 .
\end{array}
$$

From (HLY4), we have

$$
\begin{aligned}
& {\left[\left[x_{1}, x_{2}\right]_{\lambda}, \alpha\left(x_{3}\right), \alpha\left(y_{1}\right)\right]_{\lambda}+c . p . } \\
= & {\left[\left[x_{1}, x_{2}\right], \alpha\left(x_{3}\right), \alpha\left(y_{1}\right)\right]+c . p } \\
& \lambda\left\{\omega\left(\left[x_{1}, x_{2}\right], \alpha\left(x_{3}\right), \alpha\left(y_{1}\right)\right)+c . p .+\left[\nu\left(x_{1}, x_{2}\right), \alpha\left(x_{3}\right), \alpha\left(y_{1}\right)\right]+c . p \cdot\right\} \\
& +\lambda^{2}\left\{\omega\left(\nu\left(x_{1}, x_{2}\right), \alpha\left(x_{3}\right), \alpha\left(y_{1}\right)\right)+c . p \cdot\right\} \\
= & 0,
\end{aligned}
$$

thus we get

$$
\begin{array}{r}
\omega\left(\left[x_{1}, x_{2}\right], \alpha\left(x_{3}\right), \alpha\left(y_{1}\right)\right)+c . p .+\left[\nu\left(x_{1}, x_{2}\right), \alpha\left(x_{3}\right), \alpha\left(y_{1}\right)\right]+c . p .=0, \\
\omega\left(\nu\left(x_{1}, x_{2}\right), \alpha\left(x_{3}\right), \alpha\left(y_{1}\right)\right)+c . p .=0 .
\end{array}
$$

From (HLY5), we have

$$
\left[\alpha\left(x_{1}\right), \alpha\left(x_{2}\right),\left[y_{1}, y_{2}\right]_{\lambda}\right]_{\lambda}=\left[\left[x_{1}, x_{2}, y_{1}\right]_{\lambda}, \alpha^{2}\left(y_{2}\right)\right]_{\lambda}+\left[\alpha^{2}\left(y_{1}\right),\left[x_{1}, x_{2}, y_{2}\right]_{\lambda}\right]_{\lambda},
$$

the left hand side is equal to

$$
\begin{aligned}
& {\left[\alpha\left(x_{1}\right), \alpha\left(x_{2}\right),\left[y_{1}, y_{2}\right]+\lambda \nu\left(y_{1}, y_{2}\right)\right]_{\lambda} } \\
= & {\left[\alpha\left(x_{1}\right), \alpha\left(x_{2}\right),\left[y_{1}, y_{2}\right]\right] } \\
& +\lambda\left\{\omega\left(\alpha\left(x_{1}\right), \alpha\left(x_{2}\right),\left[y_{1}, y_{2}\right]\right)+\left[\alpha\left(x_{1}\right), \alpha\left(x_{2}\right), \nu\left(y_{1}, y_{2}\right)\right]\right\} \\
& +\lambda^{2} \omega\left(\alpha\left(x_{1}\right), \alpha\left(x_{2}\right), \nu\left(y_{1}, y_{2}\right)\right),
\end{aligned}
$$

and the right hand side is equal to

$$
\begin{aligned}
& {\left[\left[x_{1}, x_{2}, y_{1}\right]_{\lambda}, \alpha^{2}\left(y_{2}\right)\right]_{\lambda}+\left[\alpha^{2}\left(y_{1}\right),\left[x_{1}, x_{2}, y_{2}\right]_{\lambda}\right]_{\lambda} } \\
= & {\left[\left[x_{1}, x_{2}, y_{1}\right], \alpha^{2}\left(y_{2}\right)\right]+\left[\alpha^{2}\left(y_{1}\right),\left[x_{1}, x_{2}, y_{2}\right]\right] } \\
& +\lambda\left\{\left[\omega\left(x_{1}, x_{2}, y_{1}\right), \alpha^{2}\left(y_{2}\right)\right]+\nu\left(\left[x_{1}, x_{2}, y_{1}\right], \alpha^{2}\left(y_{2}\right)\right)\right. \\
& +\left[\alpha^{2}\left(y_{1}\right), \omega\left(x_{1}, x_{2}, y_{2}\right]+\nu\left(y_{1},\left[x_{1}, x_{2}, y_{2}\right]\right)\right\} \\
& +\lambda^{2}\left\{\nu\left(\omega\left(x_{1}, x_{2}, y_{1}\right), \alpha^{2}\left(y_{2}\right)\right)+\nu\left(\alpha^{2}\left(y_{1}\right), \omega\left(x_{1}, x_{2}, y_{2}\right)\right)\right\},
\end{aligned}
$$

then we obtain

$$
\begin{aligned}
& \omega\left(\alpha\left(x_{1}\right), \alpha\left(x_{2}\right),\left[y_{1}, y_{2}\right]\right)+\left[\alpha\left(x_{1}\right), \alpha\left(x_{2}\right), \nu\left(y_{1}, y_{2}\right)\right] \\
= & {\left[\omega\left(\alpha\left(x_{1}\right), \alpha\left(x_{2}\right), y_{1}\right), \alpha^{2}\left(y_{2}\right)\right]+\nu\left(\left[x_{1}, x_{2}, y_{1}\right], \alpha^{2}\left(y_{2}\right)\right) } \\
& +\left[\alpha^{2}\left(y_{1}\right), \omega\left(x_{1}, x_{2}, y_{2}\right)\right]+\nu\left(\alpha^{2}\left(y_{1}\right),\left[x_{1}, x_{2}, y_{2}\right]\right),
\end{aligned}
$$

and

$$
\omega\left(\alpha\left(x_{1}\right), \alpha\left(x_{2}\right), \nu\left(y_{1}, y_{2}\right)\right)=\nu\left(\omega\left(x_{1}, x_{2}, y_{1}\right), \alpha^{2}\left(y_{2}\right)\right)+\nu\left(\alpha^{2}\left(y_{1}\right), \omega\left(x_{1}, x_{2}, y_{2}\right)\right) .
$$

From (HLY6), we have

$$
\left[\alpha^{2}\left(x_{1}\right), \alpha^{2}\left(x_{2}\right),\left[y_{1}, y_{2}, y_{3}\right]_{\lambda}\right]_{\lambda}
$$




$$
\begin{aligned}
= & {\left[\left[x_{1}, x_{2}, y_{1}\right]_{\lambda}, \alpha^{2}\left(y_{2}\right), \alpha^{2}\left(y_{3}\right)\right]_{\lambda}+\left[\alpha^{2}\left(y_{1}\right),\left[x_{1}, x_{2}, y_{2}\right]_{\lambda}, \alpha^{2}\left(y_{3}\right)\right]_{\lambda} } \\
& +\left[\alpha^{2}\left(y_{1}\right), \alpha^{2}\left(y_{2}\right),\left[x_{1}, x_{2}, y_{3}\right]_{\lambda}\right]_{\lambda},
\end{aligned}
$$

the left hand side is equal to

$$
\begin{aligned}
& {\left[\alpha^{2}\left(x_{1}\right), \alpha^{2}\left(x_{2}\right),\left[y_{1}, y_{2}, y_{3}\right]+\lambda \omega\left(y_{1}, y_{2}, y_{3}\right)\right]_{\lambda} } \\
= & {\left[\alpha^{2}\left(x_{1}\right), \alpha^{2}\left(x_{2}\right),\left[y_{1}, y_{2}, y_{3}\right]\right]+\lambda \omega\left(\alpha^{2}\left(x_{1}\right), \alpha^{2}\left(x_{2}\right),\left[y_{1}, y_{2}, y_{3}\right]\right) } \\
& +\left[\alpha^{2}\left(x_{1}\right), \alpha^{2}\left(x_{2}\right), \lambda \omega\left(y_{1}, y_{2}, y_{3}\right)\right]+\lambda \omega\left(\alpha^{2}\left(x_{1}\right), \alpha^{2}\left(x_{2}\right), \lambda \omega\left(y_{1}, y_{2}, y_{3}\right)\right) \\
= & {\left[\alpha^{2}\left(x_{1}\right), \alpha^{2}\left(x_{2}\right),\left[y_{1}, y_{2}, y_{3}\right]\right] } \\
& +\lambda\left\{\omega\left(\alpha^{2}\left(x_{1}\right), \alpha^{2}\left(x_{2}\right),\left[y_{1}, y_{2}, y_{3}\right]\right)+\left[\alpha^{2}\left(x_{1}\right), \alpha^{2}\left(x_{2}\right), \omega\left(y_{1}, y_{2}, y_{3}\right)\right]\right\} \\
& +\lambda^{2} \omega\left(\alpha^{2}\left(x_{1}\right), \alpha^{2}\left(x_{2}\right), \omega\left(y_{1}, y_{2}, y_{3}\right)\right),
\end{aligned}
$$

and the right hand side is equal to

$$
\begin{aligned}
& {\left[\left[x_{1}, x_{2}, y_{1}\right]+\lambda \omega\left(x_{1}, x_{2}, y_{1}\right), \alpha^{2}\left(y_{2}\right), \alpha^{2}\left(y_{3}\right)\right]_{\lambda} } \\
& +\left[\alpha^{2}\left(y_{1}\right),\left[x_{1}, x_{2}, y_{2}\right]+\lambda \omega\left(x_{1}, x_{2}, y_{2}\right), \alpha^{2}\left(y_{3}\right)\right]_{\lambda} \\
& +\left[\alpha^{2}\left(y_{1}\right), \alpha^{2}\left(y_{2}\right),\left[x_{1}, x_{2}, y_{3}\right]+\lambda \omega\left(x_{1}, x_{2}, y_{3}\right)\right]_{\lambda} \\
= & {\left[\left[x_{1}, x_{2}, y_{1}\right], \alpha^{2}\left(y_{2}\right), \alpha^{2}\left(y_{3}\right)\right]+\left[y_{1},\left[x_{1}, x_{2}, y_{2}\right], y_{3}\right]+\left[\alpha^{2}\left(y_{1}\right), \alpha^{2}\left(y_{2}\right),\left[x_{1}, x_{2}, y_{3}\right]\right] } \\
& +\lambda\left\{\omega\left(\left[x_{1}, x_{2}, y_{1}\right], \alpha^{2}\left(y_{2}\right), \alpha^{2}\left(y_{3}\right)\right)+\left[\omega\left(x_{1}, x_{2}, y_{1}\right), \alpha^{2}\left(y_{2}\right), \alpha^{2}\left(y_{3}\right)\right]\right. \\
& +\omega\left(\alpha^{2}\left(y_{1}\right),\left[x_{1}, x_{2}, y_{2}\right], \alpha^{2}\left(y_{3}\right)\right)+\left[\alpha^{2}\left(y_{1}\right), \omega\left(x_{1}, x_{2}, y_{2}\right), \alpha^{2}\left(y_{3}\right)\right] \\
& \left.+\omega\left(\alpha^{2}\left(y_{1}\right), \alpha^{2}\left(y_{2}\right),\left[x_{1}, x_{2}, y_{3}\right]\right)+\left[\alpha^{2}\left(y_{1}\right), \alpha^{2}\left(y_{2}\right), \omega\left(x_{1}, x_{2}, y_{3}\right)\right]\right\} \\
+ & \lambda^{2}\left\{\omega\left(\omega\left(x_{1}, x_{2}, y_{1}\right), \alpha^{2}\left(y_{2}\right), \alpha^{2}\left(y_{3}\right)\right)+\omega\left(y_{1}, \omega\left(x_{1}, x_{2}, y_{2}\right), y_{3}\right)\right. \\
+ & \left.\omega\left(\alpha^{2}\left(y_{1}\right), \alpha^{2}\left(y_{2}\right), \omega\left(x_{1}, x_{2}, y_{3}\right)\right)\right\}
\end{aligned}
$$

then we get

$$
\begin{aligned}
& \omega\left(\alpha^{2}\left(x_{1}\right), \alpha^{2}\left(x_{2}\right),\left[y_{1}, y_{2}, y_{3}\right]\right)+\left[\alpha^{2}\left(x_{1}\right), \alpha^{2}\left(x_{2}\right), \omega\left(y_{1}, y_{2}, y_{3}\right)\right] \\
= & \omega\left(\left[x_{1}, x_{2}, y_{1}\right], \alpha^{2}\left(y_{2}\right), \alpha^{2}\left(y_{3}\right)\right)+\omega\left(\alpha^{2}\left(y_{1}\right),\left[x_{1}, x_{2}, y_{2}\right], \alpha^{2}\left(y_{3}\right)\right) \\
& +\omega\left(\alpha^{2}\left(y_{1}\right), \alpha^{2}\left(y_{2}\right),\left[x_{1}, x_{2}, y_{3}\right]\right)+\left[\omega\left(x_{1}, x_{2}, y_{1}\right), \alpha^{2}\left(y_{2}\right), \alpha^{2}\left(y_{3}\right)\right] \\
& +\left[\alpha^{2}\left(y_{1}\right), \omega\left(x_{1}, x_{2}, y_{2}\right), \alpha^{2}\left(y_{3}\right)\right]+\left[\alpha^{2}\left(y_{1}\right), \alpha^{2}\left(y_{2}\right), \omega\left(x_{1}, x_{2}, y_{3}\right)\right],
\end{aligned}
$$

and

$$
\begin{aligned}
& \omega\left(\alpha^{2}\left(x_{1}\right), \alpha^{2}\left(x_{2}\right), \omega\left(y_{1}, y_{2}, y_{3}\right)\right) \\
= & \omega\left(\omega\left(x_{1}, x_{2}, y_{1}\right), \alpha^{2}\left(y_{2}\right), \alpha^{2}\left(y_{3}\right)\right)+\omega\left(y_{1}, \omega\left(x_{1}, x_{2}, y_{2}\right), y_{3}\right) \\
& +\omega\left(\alpha^{2}\left(y_{1}\right), \alpha^{2}\left(y_{2}\right), \omega\left(x_{1}, x_{2}, y_{3}\right)\right) .
\end{aligned}
$$

Therefore by (11), (12), (14), (16), (18) and (20), $(\nu, \omega)$ defines a HLYA of deformation type on $T$. Furthermore, by (11), (12), (13), (15), (17) and (19), we obtain that $(\nu, \omega)$ is a $(2,3)$-cocycle of $T$ with coefficients in the adjoint representation.

A deformation is said to be trivial if there exists a linear map $N: T \rightarrow T$ such that for $\varphi_{\lambda}=\operatorname{id}+\lambda N: T_{\lambda} \rightarrow T$ there hold

$$
\varphi_{\lambda}\left[x_{1}, x_{2}\right]_{\lambda}=\left[\varphi_{\lambda} x_{1}, \varphi_{\lambda} x_{2}\right] \text { and } \varphi_{\lambda}\left[x_{1}, x_{2}, x_{3}\right]_{\lambda}=\left[\varphi_{\lambda} x_{1}, \varphi_{\lambda} x_{2}, \varphi_{\lambda} x_{3}\right] .
$$

It follows from (21) that $N$ must satisfy the following condition

$$
N\left[N x_{1}, x_{2}\right]+N\left[x_{1}, N x_{2}\right]-N^{2}\left[x_{1}, x_{2}\right]=\left[N x_{1}, N x_{2}\right] ;
$$

and

$$
\begin{aligned}
& N\left[N x_{1}, x_{2}, x_{3}\right]+N\left[x_{1}, N x_{2}, x_{3}\right]+N\left[x_{1}, x_{2}, N x_{3}\right]-N^{2}\left[x_{1}, x_{2}, x_{3}\right] \\
= & {\left[N x_{1}, N x_{2}, x_{3}\right]+\left[N x_{1}, x_{2}, N x_{3}\right]+\left[x_{1}, N x_{2}, N x_{3}\right] . }
\end{aligned}
$$


Definition 3.2. A linear operator $N: T \rightarrow T$ is called a Nijenhuis operator of a HLYA $T$ if (22) and (23) hold.

An important property of Nijenhuis operator is that it gives trivial deformation.

Theorem 3.3. Let $N$ be a Nijenhuis operator for $T$. Then a deformation of $T$ can be obtained by putting

$$
\begin{aligned}
\nu\left(x_{1}, x_{2}\right) & =\left[N x_{1}, x_{2}\right]+\left[x_{1}, N x_{2}\right]-N\left[x_{1}, x_{2}\right], \\
\omega\left(x_{1}, x_{2}, x_{3}\right) & =\left[N x_{1}, x_{2}, x_{3}\right]+\left[x_{1}, N x_{2}, x_{3}\right]+\left[x_{1}, x_{2}, N x_{3}\right]-N\left[x_{1}, x_{2}, x_{3}\right] .
\end{aligned}
$$

Furthermore, this deformation is a trivial one.

\section{Abelian Extensions}

In this section, we study abelian extensions of HLYAs. It is showed that abelian extensions are classified by the (2,3)-cohomology group. We will built a bijection map from the set of equivalent classes of abelian extensions $\operatorname{Ext}(T, V)$ and $H^{2}(T, V) \times H^{3}(T, V)$.

Definition 4.1. Let $(T,[\cdot, \cdot],[\cdot, \cdot, \cdot], \alpha),\left(V,[\cdot, \cdot]_{V},[\cdot, \cdot, \cdot]_{V}, \beta\right)$ and $\left(\hat{T},[\cdot, \cdot]_{\hat{T}},[\cdot, \cdot, \cdot]_{\hat{T}}, \hat{\alpha}\right)$ be HLYAs, $i: V \rightarrow \hat{T}, p: \hat{T} \rightarrow T$ be homomorphisms. If the following diagram commutes and the horizontal two lines are short exact sequence (i.e. $\operatorname{Im}(i)=\operatorname{Ker}(p), \operatorname{Ker}(i)=0$ and $\operatorname{Im}(p)=T)$,

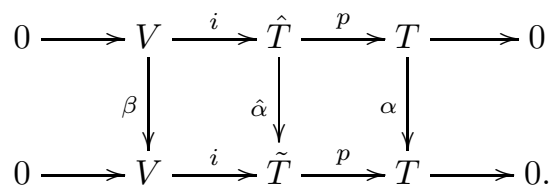

then we call $\hat{T}$ an extension of $T$ through $V$, and denote it by $\mathrm{E}_{\hat{T}}$. It is called an abelian extension if $V$ is abelian ideal of $\hat{T}$, i.e. $[u, v]_{\hat{T}}=0$ and $[u, v, \cdot]_{\hat{T}}=[u, \cdot, v]_{\hat{T}}=[\cdot, u, v]_{\hat{T}}=0$, for all $u, v \in V$.

From the left square in the commutative diagram we deduce that if we choose element $u \in V$, then

$$
\hat{\alpha} \circ i(u)=i \circ \alpha_{v}(u) .
$$

Since $i$ is a injective map, we can identify $V$ with its image in $\hat{T}$, thus we have

$$
\hat{\alpha}(u)=\left.\hat{\alpha}\right|_{V}(u)=\beta(u) .
$$

A section $\sigma: T \rightarrow \hat{T}$ of $p: \hat{T} \rightarrow T$ consists of linear maps $\sigma: T \rightarrow \hat{T}$ such that

$$
p \circ \sigma=\operatorname{id}_{T} \text { and } \hat{\alpha} \circ \sigma=\sigma \circ \alpha .
$$

Definition 4.2. Two extensions of HLYAs $\mathrm{E}_{\hat{T}}: 0 \rightarrow V \stackrel{i}{\rightarrow} \hat{T} \stackrel{p}{\rightarrow} T \rightarrow 0$ and $\mathrm{E}_{\tilde{T}}: 0 \rightarrow V \stackrel{j}{\rightarrow} \tilde{T} \stackrel{q}{\rightarrow}$ $T \rightarrow 0$ are called equivalent, if there exists a HLYA homomorphism $F: \hat{T} \rightarrow \tilde{T}$ such that the following diagram commutes

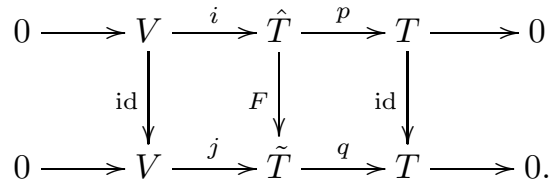

The set of equivalent classes of extensions of $T$ by $V$ is denoted by $\operatorname{Ext}(T, V)$. 
Let $\hat{T}$ be an abelian extension of $T$ by $V$. Define maps $\rho$ from $T$ to $\operatorname{End}(V)$ and $D, \theta$ from $T \times T$ to $\operatorname{End}(V)$ by

$$
\begin{aligned}
\rho\left(x_{1}\right)(u) & \triangleq\left[\sigma\left(x_{1}\right), u\right]_{\hat{T}}, \\
D\left(x_{1}, x_{2}\right)(u) & \triangleq\left[\sigma\left(x_{1}\right), \sigma\left(x_{2}\right), u\right]_{\hat{T}}, \\
\theta\left(x_{1}, x_{2}\right)(u) & \triangleq\left[u, \sigma\left(x_{1}\right), \sigma\left(x_{2}\right)\right]_{\hat{T}} .
\end{aligned}
$$

Lemma 4.3. With the above notations, $(\rho, D, \theta)$ is a representation of $T$ on $V$ and does not depend on the choice of the section $\sigma$. Moreover, equivalent abelian extensions give the same representation.

Proof. First, the fact that $\rho, D, \theta$ are independent of the choice of $\sigma$ is easy to check. For details, see 21.

Second, we will show that $(\rho, D, \theta)$ is a representation of $T$ on $V$.

By the equality

$$
\begin{aligned}
\rho\left(\alpha\left(x_{1}\right)\right) \circ \beta(u) & =\left[\sigma\left(\alpha\left(x_{1}\right)\right), \beta(u)\right]_{\hat{T}} \\
& =\left[\hat{\alpha} \circ \sigma\left(x_{1}\right), \hat{\alpha}(u)\right]_{\hat{T}} \\
& =\hat{\alpha}\left(\left[\sigma\left(x_{1}\right), u\right]_{\hat{T}}\right) \\
& =\beta\left(\left[\sigma\left(x_{1}\right), u\right]_{\hat{T}}\right)=\beta \circ \rho\left(x_{1}\right)(u),
\end{aligned}
$$

we obtain (HR01):

$$
\rho\left(\alpha\left(x_{1}\right)\right) \circ \beta=\beta \circ \rho\left(x_{1}\right) .
$$

By the equality

$$
\begin{aligned}
D\left(\alpha\left(x_{1}\right), \alpha\left(x_{2}\right)\right) \circ \beta(u) & =\left[\sigma\left(\alpha\left(x_{1}\right)\right), \sigma\left(\alpha\left(x_{2}\right)\right), \beta(u)\right]_{\hat{T}} \\
& =\left[\hat{\alpha} \circ \sigma\left(x_{1}\right), \hat{\alpha} \circ \sigma\left(x_{2}\right), \hat{\alpha}(u)\right]_{\hat{T}} \\
& =\hat{\alpha}\left(\left[\sigma\left(x_{1}\right), \sigma\left(x_{2}\right), u\right]_{\hat{T}}\right) \\
& =\beta\left(\left[\sigma\left(x_{1}\right), \sigma\left(x_{2}\right), u\right]_{\hat{T}}\right)=\beta \circ D\left(x_{1}, x_{2}\right)(u),
\end{aligned}
$$

we obtain (HR02):

$$
D\left(\alpha\left(x_{1}\right), \alpha\left(x_{2}\right)\right) \circ \beta(u)=\beta \circ D\left(x_{1}, x_{2}\right)(u) .
$$

By the equality

$$
\begin{aligned}
& {\left[\sigma\left(x_{1}\right), \sigma\left(x_{2}\right), u\right]_{\hat{T}}+\left[\sigma\left(x_{2}\right), u, \sigma\left(x_{1}\right)\right]_{\hat{T}}+\left[u, \sigma\left(x_{1}\right), \sigma\left(x_{2}\right)\right]_{\hat{T}}} \\
& +\left[\left[\sigma\left(x_{1}\right), \sigma\left(x_{2}\right)\right]_{\hat{T}}, \beta(u)\right]_{\hat{T}}+\left[\left[\sigma\left(x_{2}\right), u\right]_{\hat{T}}, \hat{\alpha} \circ \sigma\left(x_{1}\right)\right]_{\hat{T}}+\left[\left[u, \sigma\left(x_{1}\right)\right], \hat{\alpha} \circ \sigma\left(x_{2}\right)\right]_{\hat{T}}=0,
\end{aligned}
$$

we obtain (HR31):

$$
D\left(x_{1}, x_{2}\right)-\theta\left(x_{2}, x_{1}\right)+\theta\left(x_{1}, x_{2}\right)+\rho\left(\left[x_{1}, x_{2}\right]\right) \circ \beta-\rho\left(\alpha\left(x_{1}\right)\right) \rho\left(x_{2}\right)+\rho\left(\alpha\left(x_{2}\right)\right) \rho\left(x_{1}\right)=0 .
$$

By the equality

$$
\begin{aligned}
& {\left[\left[\sigma\left(x_{1}\right), \sigma\left(x_{2}\right)\right]_{\hat{T}}, \hat{\alpha} \circ \sigma\left(x_{3}\right), \beta(u)\right]_{\hat{T}}+\left[\left[\sigma\left(x_{2}\right), \sigma\left(x_{3}\right)\right]_{\hat{T}}, \hat{\alpha} \circ \sigma\left(x_{1}\right), \beta(u)\right]_{\hat{T}}} \\
& +\left[\left[\sigma\left(x_{3}\right), \sigma\left(x_{1}\right)\right]_{\hat{T}}, \hat{\alpha} \circ \sigma\left(x_{2}\right), \beta(u)\right]_{\hat{T}}=0,
\end{aligned}
$$

we have (HR41):

$$
D\left(\left[x_{1}, x_{2}\right], \alpha\left(x_{3}\right)\right)+D\left(\left[x_{2}, x_{3}\right], \alpha\left(x_{1}\right)\right)+D\left(\left[x_{3}, x_{1}\right], \alpha\left(x_{2}\right)\right)=0 .
$$


By the equality

$$
\begin{aligned}
& {\left[\left[\sigma\left(x_{1}\right), \sigma\left(x_{2}\right)\right]_{\hat{T}}, \beta(u), \hat{\alpha} \circ \sigma\left(y_{1}\right)\right]_{\hat{T}}+\left[\left[\sigma\left(x_{2}\right), u\right]_{\hat{T}}, \hat{\alpha} \circ \sigma\left(x_{1}\right), \hat{\alpha} \circ \sigma\left(y_{1}\right)\right]_{\hat{T}}} \\
& +\left[\left[u, \sigma\left(x_{1}\right)\right]_{\hat{T}}, \hat{\alpha} \circ \sigma\left(x_{2}\right), \hat{\alpha} \circ \sigma\left(y_{1}\right)\right]_{\hat{T}}=0,
\end{aligned}
$$

we have (HR42):

$$
\theta\left(\left[x_{1}, x_{2}\right], \alpha\left(y_{1}\right)\right) \circ \beta=\theta\left(\alpha\left(x_{1}\right), \alpha\left(y_{1}\right)\right) \rho\left(x_{2}\right)-\theta\left(\alpha\left(x_{2}\right), \alpha\left(y_{1}\right)\right) \rho\left(x_{1}\right) .
$$

By the equality

$$
\begin{aligned}
{\left[\hat{\alpha} \circ \sigma\left(x_{1}\right), \hat{\alpha} \circ \sigma\left(x_{2}\right),\left[u, \sigma\left(y_{2}\right)\right]_{\hat{T}}\right]_{\hat{T}}=} & {\left[\left[\sigma\left(x_{1}\right), \sigma\left(x_{2}\right), u\right]_{\hat{T}}, \hat{\alpha}^{2} \circ \sigma\left(y_{2}\right)\right]_{\hat{T}} } \\
& +\left[\beta^{2}(u),\left[\sigma\left(x_{1}\right), \sigma\left(x_{2}\right), \sigma\left(y_{2}\right)\right]_{\hat{T}}\right]_{\hat{T}},
\end{aligned}
$$

we have (HR51):

$$
D\left(\alpha\left(x_{1}\right), \alpha\left(x_{2}\right)\right) \rho\left(y_{2}\right)=\rho\left(\alpha^{2}\left(y_{2}\right)\right) D\left(x_{1}, x_{2}\right)+\rho\left(\left[x_{1}, x_{2}, y_{2}\right]\right) \circ \beta^{2} .
$$

By the equality

$$
\begin{aligned}
{\left[\hat{\alpha} \circ \sigma\left(x_{1}\right), \beta(u),\left[\sigma\left(y_{1}\right), \sigma\left(y_{2}\right)\right]_{\hat{T}}\right]_{\hat{T}}=} & {\left[\left[\sigma\left(x_{1}\right), u, \sigma\left(y_{1}\right)\right]_{\hat{T}}, \hat{\alpha}^{2} \circ \sigma\left(y_{2}\right)\right]_{\hat{T}} } \\
& +\left[\hat{\alpha}^{2} \circ \sigma\left(y_{1}\right),\left[\sigma\left(x_{1}\right), u, \sigma\left(y_{2}\right)\right]_{\hat{T}}\right]_{\hat{T}},
\end{aligned}
$$

we have (HR52):

$$
\theta\left(\alpha\left(x_{1}\right),\left[y_{1}, y_{2}\right]\right) \circ \beta=\rho\left(\alpha^{2}\left(y_{1}\right)\right) \theta\left(x_{1}, y_{2}\right)-\rho\left(\alpha^{2}\left(y_{2}\right)\right) \theta\left(x_{1}, y_{1}\right) .
$$

By the equality

$$
\begin{aligned}
& {\left[\hat{\alpha}^{2} \circ \sigma\left(x_{1}\right), \hat{\alpha}^{2} \circ \sigma\left(x_{2}\right),\left[u, \sigma\left(y_{1}\right), \sigma\left(y_{2}\right)\right]_{\hat{T}}\right]_{\hat{T}} } \\
= & {\left[\left[\sigma\left(x_{1}\right), \sigma\left(x_{2}\right), u\right]_{\hat{T}}, \hat{\alpha}^{2} \circ \sigma\left(y_{1}\right), \hat{\alpha}^{2} \circ \sigma\left(y_{2}\right)\right]_{\hat{T}}+\left[\beta^{2}(u),\left[\sigma\left(x_{1}\right), \sigma\left(x_{2}\right), \sigma\left(y_{1}\right)\right]_{\hat{T}}, \hat{\alpha}^{2} \circ \sigma\left(y_{2}\right)\right]_{\hat{T}} } \\
& +\left[\beta^{2}(u), \hat{\alpha}^{2} \circ \sigma\left(y_{1}\right),\left[\sigma\left(x_{1}\right), \sigma\left(x_{2}\right), \sigma\left(y_{2}\right)\right]_{\hat{T}}\right]_{\hat{T}},
\end{aligned}
$$

we have (HR61):

$$
\begin{aligned}
D\left(\alpha^{2}\left(x_{1}\right), \alpha^{2}\left(x_{2}\right)\right) \theta\left(y_{1}, y_{2}\right)= & \theta\left(\alpha^{2}\left(y_{1}\right), \alpha^{2}\left(y_{2}\right)\right) D\left(x_{1}, x_{2}\right)+\theta\left(\left[x_{1}, x_{2}, y_{1}\right], \alpha^{2}\left(y_{2}\right)\right) \circ \beta^{2} \\
& +\theta\left(\alpha^{2}\left(y_{1}\right),\left[x_{1}, x_{2}, y_{2}\right]\right) \circ \beta^{2} .
\end{aligned}
$$

By the equality

$$
\begin{aligned}
& {\left[\beta^{2}(u), \hat{\alpha}^{2} \circ \sigma\left(x_{1}\right),\left[\sigma\left(y_{1}\right), \sigma\left(y_{2}\right), \sigma\left(y_{3}\right)\right]_{\hat{T}}\right]_{\hat{T}} } \\
= & {\left[\left[u, \sigma\left(x_{1}\right), \sigma\left(y_{1}\right)\right]_{\hat{T}}, \hat{\alpha}^{2} \circ \sigma\left(y_{2}\right), \hat{\alpha}^{2} \circ \sigma\left(y_{3}\right)\right]_{\hat{T}}+\left[\hat{\alpha}^{2} \circ \sigma\left(y_{1}\right),\left[u, \sigma\left(x_{1}\right), \sigma\left(y_{2}\right)\right]_{\hat{T}}, \hat{\alpha}^{2} \circ \sigma\left(y_{3}\right)\right]_{\hat{T}} } \\
+ & {\left[\hat{\alpha}^{2} \circ \sigma\left(y_{1}\right), \hat{\alpha}^{2} \circ \sigma\left(y_{2}\right),\left[u, \sigma\left(x_{1}\right), \sigma\left(y_{3}\right)\right]_{\hat{T}}\right]_{\hat{T}}, }
\end{aligned}
$$

we have (HR62):

$$
\begin{aligned}
\theta\left(\alpha^{2}\left(x_{1}\right),\left[y_{1}, y_{2}, y_{3}\right]\right) \circ \beta^{2}= & \theta\left(\alpha^{2}\left(y_{2}\right), \alpha^{2}\left(y_{3}\right)\right) \theta\left(x_{1}, y_{1}\right)-\theta\left(\alpha^{2}\left(y_{1}\right), \alpha^{2}\left(y_{3}\right)\right) \theta\left(x_{1}, y_{2}\right) \\
& +D\left(\alpha^{2}\left(y_{1}\right), \alpha^{2}\left(y_{2}\right)\right) \theta\left(x_{1}, y_{3}\right) .
\end{aligned}
$$

Therefore we see that $(\rho, D, \theta)$ is a representation of $T$ on $V$.

At last, suppose that $\mathrm{E}_{\hat{T}}$ and $\mathrm{E}_{\tilde{T}}$ are equivalent abelian extensions, and $F: \hat{T} \rightarrow \tilde{T}$ is the HLYA homomorphism satisfying $F \circ i=j, q \circ F=p$. Choose linear sections $\sigma$ and $\sigma^{\prime}$ of $p$ and $q$, we get $q F \sigma\left(x_{i}\right)=p \sigma\left(x_{i}\right)=x_{i}=q \sigma^{\prime}\left(x_{i}\right)$, then $F \sigma\left(x_{i}\right)-\sigma^{\prime}\left(x_{i}\right) \in \operatorname{Ker}(q) \cong V$. Thus, we have

$$
\left[u, \sigma\left(x_{1}\right), \sigma\left(x_{2}\right)\right]_{\hat{T}}=\left[u, F \sigma\left(x_{1}\right), F \sigma\left(x_{2}\right)\right]_{\tilde{T}}=\left[u, \sigma^{\prime}\left(x_{1}\right), \sigma^{\prime}\left(x_{2}\right)\right]_{\tilde{T}}
$$


Therefore, equivalent abelian extensions give the same $\theta$. Similarly, one can prove that equivalent abelian extensions give the same $D$ and $\rho$. The proof is finished.

Let $\sigma: T \rightarrow \hat{T}$ be a section of abelian extension. Define the following maps:

$$
\begin{aligned}
\nu\left(x_{1}, x_{2}\right) & \triangleq\left[\sigma\left(x_{1}\right), \sigma\left(x_{2}\right)\right]_{\hat{T}}-\sigma\left(\left[x_{1}, x_{2}\right]\right), \\
\omega\left(x_{1}, x_{2}, x_{3}\right) & \triangleq\left[\sigma\left(x_{1}\right), \sigma\left(x_{2}\right), \sigma\left(x_{3}\right)\right]_{\hat{T}}-\sigma\left(\left[x_{1}, x_{2}, x_{3}\right]\right),
\end{aligned}
$$

Lemma 4.4. Let $0 \rightarrow V \rightarrow \hat{T} \rightarrow T \rightarrow 0$ be an abelian extension of $T$ by $V$. Then $(\nu, \omega)$ defined by (43) and (44) is a $(2,3)$-cocycle of $T$ with coefficients in $V$.

Proof. First, we claim that the image of $\nu$ is contained in $V$, that is to say, $p \circ \nu\left(x_{1}, x_{2}\right)=0$. In fact, since $p$ is an algebraic homomorphism, we have

$$
p \circ \nu\left(x_{1}, x_{2}\right)=\left[p \circ \sigma\left(x_{1}\right), p \circ \sigma\left(x_{2}\right)\right]_{\hat{T}}-p \circ \sigma\left(\left[x_{1}, x_{2}\right]\right)=0
$$

Next, one check that $\nu$ and $\omega$ defined above satisfies (CC01) and (CC02). For example

$$
\begin{aligned}
& \nu\left(\alpha\left(x_{1}\right), \alpha\left(x_{2}\right)\right) \\
= & {\left[\sigma\left(\alpha\left(x_{1}\right)\right), \sigma\left(\alpha\left(x_{2}\right)\right)\right]_{\hat{T}}-\sigma\left(\left[\alpha\left(x_{1}\right), \alpha\left(x_{2}\right)\right]\right) } \\
= & {\left[\sigma \circ \alpha\left(x_{1}\right), \sigma \circ \alpha\left(x_{2}\right)\right]_{\hat{T}}-\sigma \circ \alpha\left(\left[x_{1}, x_{2}\right]\right) } \\
= & \left(\left[\hat{\alpha} \circ \sigma\left(x_{1}\right), \hat{\alpha} \circ \sigma\left(x_{2}\right)\right]_{\hat{T}}\right)-\hat{\alpha} \circ \sigma\left(\left[x_{1}, x_{2}\right]\right) \\
= & \left.\hat{\alpha}\left(\left[\sigma\left(x_{1}\right), \sigma\left(x_{2}\right)\right]_{\hat{T}}\right)-\sigma\left(\left[x_{1}, x_{2}\right]\right)\right) \\
= & \beta\left(\left[\sigma\left(x_{1}\right), \sigma\left(x_{2}\right)\right]_{\hat{T}}-\sigma\left(\left[x_{1}, x_{2}\right]\right)\right) \\
= & \beta\left(\nu\left(x_{1}, x_{2}\right)\right) .
\end{aligned}
$$

Finally, we verify that $\nu$ and $\omega$ satisfies $(\mathrm{CC} 1)-(\mathrm{CC} 4)$.

By the equality

$$
\left[\sigma x_{1}, \sigma x_{2}, \sigma x_{3}\right]_{\hat{T}}+c . p .+\left[\left[\sigma x_{1}, \sigma x_{2}\right]_{\hat{T}}, \hat{\alpha}\left(\sigma\left(x_{3}\right)\right)\right]_{\hat{T}}+c . p .=0
$$

we obtain that

$$
\begin{aligned}
& \left\{\omega\left(\left[x_{1}, x_{2}, x_{3}\right)+\sigma\left[x_{1}, x_{2}, x_{3}\right]_{\hat{T}}\right\}+\right.\text { c.p. } \\
& +\left\{\left[\nu\left(x_{1}, x_{2}\right), \sigma\left(\alpha\left(x_{3}\right)\right)\right]_{\hat{T}}+\nu\left(\left[x_{1}, x_{2}\right], \alpha\left(x_{3}\right)\right)+\sigma\left(\left[\left[x_{1}, x_{2}\right], \alpha\left(x_{3}\right)\right]\right)\right\}+\text { c.p. }=0 .
\end{aligned}
$$

Thus we have (CC1):

$$
\omega\left(x_{1}, x_{2}, x_{3}\right)+c . p .-\rho\left(\alpha\left(x_{3}\right)\right) \nu\left(x_{1}, x_{2}\right)-c . p .+\nu\left(\left[x_{1}, x_{2}\right], \alpha\left(x_{3}\right)\right)+c . p .=0 .
$$

By the equality

$$
\left[\left[\sigma x_{1}, \sigma x_{2}\right]_{\hat{T}}, \hat{\alpha}\left(\sigma x_{3}\right), \hat{\alpha}\left(\sigma y_{1}\right)\right]_{\hat{T}}+c . p .=0,
$$

we get

$$
\left\{\left[\nu\left(x_{1}, x_{2}\right), \sigma \alpha\left(x_{3}\right), \sigma \alpha\left(y_{1}\right)\right]_{\hat{T}}+\omega\left(\left[x_{1}, x_{2}\right], \alpha\left(x_{3}\right), \alpha\left(y_{1}\right)\right)+\sigma\left[\left[x_{1}, x_{2}\right], \alpha\left(x_{3}\right), \alpha\left(y_{1}\right)\right]\right\}+c . p .=0 .
$$

Thus we have (CC2):

$$
\theta\left(\alpha\left(x_{3}\right), \alpha\left(y_{1}\right)\right) \nu\left(x_{1}, x_{2}\right)+c . p .+\omega\left(\left[x_{1}, x_{2}\right], \alpha\left(x_{3}\right), \alpha\left(y_{1}\right)\right)+c . p .=0 .
$$

By the equality

$$
\left[\hat{\alpha}\left(\sigma x_{1}\right), \hat{\alpha}\left(\sigma x_{2}\right),\left[\sigma y_{1}, \sigma y_{2}\right]_{\hat{T}}\right]_{\hat{T}}
$$




$$
=\left[\left[\sigma x_{1}, \sigma x_{2}, \sigma y_{1}\right]_{\hat{T}}, \hat{\alpha}^{2}\left(\sigma y_{2}\right)\right]_{\hat{T}}+\left[\hat{\alpha}^{2}\left(\sigma y_{1}\right),\left[\sigma x_{1}, \sigma x_{2}, \sigma y_{2}\right]_{\hat{T}}\right]_{\hat{T}},
$$

we obtain that the left hand side is equal to

$$
\begin{aligned}
& {\left[\sigma \alpha\left(x_{1}\right), \sigma \alpha\left(x_{2}\right),\left[\sigma y_{1}, \sigma y_{2},\right]_{\hat{T}}\right]_{\hat{T}} } \\
= & {\left[\sigma \alpha\left(x_{1}\right), \sigma \alpha\left(x_{2}\right), \nu\left(y_{1}, y_{2}\right)+\sigma\left(\left[y_{1}, y_{2}\right]_{T}\right)\right]_{\hat{T}} } \\
= & D\left(\alpha\left(x_{1}\right), \alpha\left(x_{2}\right)\right) \nu\left(y_{1}, y_{2}\right)+\left[\sigma \alpha\left(x_{1}\right), \sigma \alpha\left(x_{2}\right), \sigma\left(\left[y_{1}, y_{2}\right]\right)\right]_{\hat{T}} \\
= & D\left(\alpha\left(x_{1}\right), \alpha\left(x_{2}\right)\right) \nu\left(y_{1}, y_{2}\right)+\omega\left(\alpha\left(x_{1}\right), \alpha\left(x_{2}\right),\left[y_{1}, y_{2}\right]\right)+\sigma\left(\left[\alpha\left(x_{1}\right), \alpha\left(x_{2}\right),\left[y_{1}, y_{2}\right]\right]\right) .
\end{aligned}
$$

Similarily, the right hand side is equal to

$$
\begin{aligned}
& {\left[\left[\sigma x_{1}, \sigma x_{2}, \sigma y_{1}\right]_{\hat{T}}, \sigma \alpha^{2}\left(y_{2}\right)\right]_{\hat{T}}+\left[\sigma \alpha^{2}\left(y_{1}\right),\left[\sigma x_{1}, \sigma x_{2}, \sigma y_{2}\right]_{\hat{T}}\right]_{\hat{T}} } \\
= & {\left[\omega\left(x_{1}, x_{2}, y_{1}\right)+\sigma\left[x_{1}, x_{2}, y_{1}\right], \sigma y_{2}\right]_{\hat{T}}+\nu\left(\left[x_{1}, x_{2}, y_{1}\right], y_{2}\right)+\sigma\left[\left[x_{1}, x_{2}, y_{1}\right], y_{2}\right] } \\
= & -\rho\left(\alpha^{2}\left(y_{2}\right)\right) \omega\left(x_{1}, x_{2}, y_{1}\right)+\nu\left(\left[x_{1}, x_{2}, y_{1}\right], \alpha^{2}\left(y_{2}\right)\right)+\sigma\left(\left[\left[x_{1}, x_{2}, y_{1}\right], \alpha^{2}\left(y_{2}\right)\right]\right) \\
& \rho\left(\alpha^{2}\left(y_{1}\right)\right) \omega\left(x_{1}, x_{2}, y_{2}\right)+\nu\left(\alpha^{2}\left(y_{1}\right),\left[x_{1}, x_{2}, y_{2}\right]\right)+\sigma\left(\left[\alpha^{2}\left(y_{1}\right),\left[x_{1}, x_{2}, y_{2}\right]\right]\right) .
\end{aligned}
$$

Thus we have (CC3):

$$
\begin{aligned}
& \omega\left(\alpha\left(x_{1}\right), \alpha\left(x_{2}\right),\left[y_{1}, y_{2}\right]\right)+D\left(\alpha\left(x_{1}\right), \alpha\left(x_{2}\right)\right) \omega\left(y_{1}, y_{2}\right) \\
= & \left.\nu\left(\left[x_{1}, x_{2}, y_{1}\right], \alpha^{2}\left(y_{2}\right)\right)+\nu\left(\alpha^{2}\left(y_{1}\right),\left[x_{1}, x_{2}, y_{2}\right]\right)\right) \\
& +\rho\left(\alpha^{2}\left(y_{1}\right)\right) \omega\left(x_{1}, x_{2}, y_{2}\right)-\rho\left(\alpha^{2}\left(y_{2}\right)\right) \omega\left(x_{1}, x_{2}, y_{1}\right) .
\end{aligned}
$$

By the equality

$$
\begin{aligned}
& {\left[\hat{\alpha}^{2}\left(\sigma x_{1}\right), \hat{\alpha}^{2}\left(\sigma x_{2}\right),\left[\sigma y_{1}, \sigma y_{2}, \sigma y_{3}\right]_{\hat{T}}\right]_{\hat{T}} } \\
= & {\left[\left[\sigma x_{1}, \sigma x_{2}, \sigma y_{1}\right]_{\hat{T}}, \hat{\alpha}^{2}\left(\sigma y_{2}\right), \hat{\alpha}^{2}\left(\sigma y_{3}\right)\right]_{\hat{T}}+\left[\hat{\alpha}^{2}\left(\sigma y_{1}\right),\left[\sigma x_{1}, \sigma x_{2}, \sigma y_{2}\right]_{\hat{T}}, \hat{\alpha}^{2}\left(\sigma y_{3}\right)\right]_{\hat{T}} } \\
& +\left[\hat{\alpha}^{2}\left(\sigma y_{1}\right), \hat{\alpha}^{2}\left(\sigma y_{2}\right),\left[\sigma x_{1}, \sigma x_{2}, \sigma y_{3}\right]_{\hat{T}}\right]_{\hat{T}},
\end{aligned}
$$

we have that the left hand side is equal to

$$
\begin{aligned}
& {\left[\sigma \alpha^{2}\left(x_{1}\right), \sigma \alpha^{2}\left(x_{2}\right),\left[\sigma y_{1}, \sigma y_{2}, \sigma y_{3}\right]_{\hat{T}}\right]_{\hat{T}} } \\
= & {\left[\sigma \alpha^{2}\left(x_{1}\right), \sigma \alpha^{2}\left(x_{2}\right), \omega\left(y_{1}, y_{2}, y_{3}\right)+\sigma\left(\left[y_{1}, y_{2}, y_{3}\right] g\right)\right]_{\hat{T}} } \\
= & D\left(\alpha^{2}\left(x_{1}\right), \alpha^{2}\left(x_{2}\right)\right) \omega\left(y_{1}, y_{2}, y_{3}\right)+\left[\sigma x_{1}, \sigma x_{2}, \sigma\left(\left[y_{1}, y_{2}, y_{3}\right]\right)\right]_{\hat{T}} \\
= & D\left(\alpha^{2}\left(x_{1}\right), \alpha^{2}\left(x_{2}\right)\right) \omega\left(y_{1}, y_{2}, y_{3}\right)+\omega\left(\alpha^{2}\left(x_{1}\right), \alpha^{2}\left(x_{2}\right),\left[y_{1}, y_{2}, y_{3}\right]\right) \\
& +\sigma\left(\left[\alpha^{2}\left(x_{1}\right), \alpha^{2}\left(x_{2}\right),\left[y_{1}, y_{2}, y_{3}\right]\right]\right) .
\end{aligned}
$$

Similarily, the right hand side is equal to

$$
\begin{aligned}
& \theta\left(y_{2}, y_{3}\right) \omega\left(x_{1}, x_{2}, y_{1}\right)+\omega\left(\left[x_{1}, x_{2}, y_{1}\right], y_{2}, y_{3}\right)+\sigma\left(\left[\left[x_{1}, x_{2}, y_{1}\right], y_{2}, y_{3}\right]\right) \\
& -\theta\left(y_{1}, y_{3}\right) \omega\left(x_{1}, x_{2}, y_{2}\right)+\omega\left(y_{1},\left[x_{1}, x_{2}, y_{2}\right], y_{3}\right)+\sigma\left(\left[y_{1},\left[x_{1}, x_{2}, y_{2}\right], y_{3}\right]\right) \\
& +D\left(y_{1}, y_{2}\right) \omega\left(x_{1}, x_{2}, y_{3}\right)+\omega\left(y_{1}, y_{2},\left[x_{1}, x_{2}, y_{3}\right]\right)+\sigma\left(\left[\alpha^{2}\left(y_{1}\right), \alpha^{2}\left(y_{2}\right),\left[x_{1}, x_{2}, y_{3}\right]\right]\right) .
\end{aligned}
$$

Thus we have (CC4):

$$
\begin{aligned}
& \omega\left(\alpha^{2}\left(x_{1}\right), \alpha^{2}\left(x_{2}\right),\left[y_{1}, y_{2}, y_{3}\right]\right)+D\left(\alpha^{2}\left(x_{1}\right), \alpha^{2}\left(x_{2}\right)\right) \omega\left(y_{1}, y_{2}, y_{3}\right) \\
= & \omega\left(\left[x_{1}, x_{2}, y_{1}\right], \alpha^{2}\left(y_{2}\right), \alpha^{2}\left(y_{3}\right)\right)+\omega\left(\alpha^{2}\left(y_{1}\right),\left[x_{1}, x_{2}, y_{2}\right], \alpha^{2}\left(y_{3}\right)\right) \\
& +\omega\left(\alpha^{2}\left(y_{1}\right), \alpha^{2}\left(y_{2}\right),\left[x_{1}, x_{2}, y_{3}\right]\right)+\theta\left(\alpha^{2}\left(y_{2}\right), \alpha^{2}\left(y_{3}\right)\right) \omega\left(x_{1}, x_{2}, y_{1}\right) \\
& -\theta\left(\alpha^{2}\left(y_{1}\right), \alpha^{2}\left(y_{3}\right)\right) \omega\left(x_{1}, x_{2}, y_{2}\right)+D\left(\alpha^{2}\left(y_{1}\right), \alpha^{2}\left(y_{2}\right)\right) \omega\left(x_{1}, x_{2}, y_{3}\right) .
\end{aligned}
$$

Therefore we get all the (2,3)-cocycle conditions in Definition 2.9 .

From Lemma 4.3 and Lemma 4.4, we have obtained that abelian extensions of HLYA $T$ through $V$ give rise to a representation of $T$ on $V$ and a $(2,3)$-cocycle of $T$ with coefficients in $V$. Conversely, given a representation and a $(2,3)$-cocycle, we can obtain a HLYA structure on the space $T \oplus V$. 
Lemma 4.5. Let $T$ be a HLYA, $(\rho, D, \theta)$ is a representation of $T$ on $V$ and $(\nu, \omega)$ is a (2,3)-cocycle of $T$ with coefficients in $V$. Then $T \oplus V$ is a HLYA under the following bilinear and trilinear maps:

$$
\begin{aligned}
(\alpha+\beta)\left(x_{1}+u_{1}\right) \triangleq & \alpha\left(x_{1}\right)+\beta\left(u_{1}\right), \\
{\left[x_{1}+u_{1}, x_{2}+u_{2}\right]_{\nu} \triangleq } & {\left[x_{1}, x_{2}\right]+\nu\left(x_{1}, x_{2}\right)+\rho\left(x_{1}\right)\left(u_{2}\right)-\rho\left(x_{2}\right)\left(u_{1}\right), } \\
{\left[x_{1}+u_{1}, x_{2}+u_{2}, x_{3}+u_{3}\right]_{\omega} \triangleq } & {\left[x_{1}, x_{2}, x_{3}\right]+\omega\left(x_{1}, x_{2}, x_{3}\right)+D\left(x_{1}, x_{2}\right)\left(u_{3}\right) } \\
& -\theta\left(x_{1}, x_{3}\right)\left(u_{2}\right)+\theta\left(x_{2}, x_{3}\right)\left(u_{1}\right),
\end{aligned}
$$

This kind of HLYAs is denoted by $E_{(\nu, \omega)}=T \oplus_{(\nu, \omega)} V$.

Proof. We will verify that conditions (HLY01)-(HLY02) and (HLY1)-(HLY6) hold for maps defined on $T \oplus V$ by (49), (50) and (51).

Now condition (HLY01) becomes

$$
(\alpha+\beta)\left(\left[x_{1}+u_{1}, x_{2}+u_{2}\right]\right)=\left[(\alpha+\beta)\left(x_{1}+u_{1},\right),(\alpha+\beta)\left(x_{2}+u_{2}\right)\right] .
$$

The left hand is equal to

$$
\begin{aligned}
& (\alpha+\beta)\left(\left[x_{1}, x_{2}\right]+\nu\left(x_{1}, x_{2}\right)+\rho\left(x_{1}\right)\left(u_{2}\right)-\rho\left(x_{2}\right)\left(u_{1}\right)\right) \\
=\quad & \alpha\left(\left[x_{1}, x_{2}\right]\right)+\beta \circ \nu\left(x_{1}, x_{2}\right)+\beta \circ \rho\left(x_{1}\right)\left(u_{2}\right)-\beta \circ \rho\left(x_{2}\right)\left(u_{1}\right),
\end{aligned}
$$

and the right hand is equal to

$$
\begin{aligned}
& {\left[\alpha\left(x_{1}\right)+\beta\left(u_{1}\right), \alpha\left(x_{2}\right)+\beta\left(u_{2}\right)\right] } \\
= & {\left[\alpha\left(x_{1}\right), \alpha\left(x_{2}\right)\right]+\nu\left(\alpha\left(x_{1}\right), \alpha\left(x_{2}\right)\right)+\rho\left(\alpha\left(x_{1}\right)\right) \circ \beta\left(u_{2}\right)-\rho\left(\alpha\left(x_{2}\right)\right) \circ \beta\left(u_{1}\right) . }
\end{aligned}
$$

Since $\alpha$ is an algebraic homomorphism and by conditions (HR01),(CC01), we obtain equality (52).

The condition (HLY02) becomes

$$
\begin{aligned}
& (\alpha+\beta)\left(\left[x_{1}+u_{1}, x_{2}+u_{2}, x_{3}+u_{3}\right]\right) \\
=\quad & {\left[(\alpha+\beta)\left(x_{1}+u_{1}\right),(\alpha+\beta)\left(x_{2}+u_{2}\right),(\alpha+\beta)\left(x_{3}+u_{3}\right)\right] . }
\end{aligned}
$$

The left hand is equal to

$$
\begin{aligned}
& (\alpha+\beta)\left(\left[x_{1}, x_{2}, x_{3}\right]+D\left(x_{1}, x_{2}\right)\left(u_{3}\right)-\theta\left(x_{1}, x_{3}\right)\left(u_{2}\right)+\theta\left(x_{2}, x_{3}\right)\left(u_{1}\right)\right) \\
= & \alpha\left(\left[x_{1}, x_{2}, x_{3}\right]\right)+\beta \circ \omega\left(x_{1}, x_{2}, x_{3}\right)+\beta \circ D\left(x_{1}, x_{2}\right)\left(u_{3}\right)-\beta \circ \theta\left(x_{1}, x_{3}\right)\left(u_{2}\right) \\
+ & \beta \circ \theta\left(x_{2}, x_{3}\right)\left(u_{1}\right),
\end{aligned}
$$

and the right hand is equal to

$$
\begin{aligned}
& {\left[\alpha\left(x_{1}\right)+\beta\left(u_{1}\right), \alpha\left(x_{2}\right)+\beta\left(u_{2}\right), \alpha\left(x_{3}\right)+\beta\left(u_{3}\right)\right] } \\
=\quad & {\left[\alpha\left(x_{1}\right), \alpha\left(x_{2}\right), \alpha\left(x_{3}\right)\right]+\omega\left(\alpha\left(x_{1}\right), \alpha\left(x_{2}\right), \alpha\left(x_{3}\right)\right)+D\left(\alpha\left(x_{1}\right), \alpha\left(x_{2}\right)\right) \circ \beta\left(u_{3}\right) } \\
& -\theta\left(\alpha\left(x_{1}\right), \alpha\left(x_{3}\right)\right) \circ \beta\left(u_{2}\right)+\theta\left(\alpha\left(x_{2}\right), \alpha\left(x_{3}\right)\right) \circ \beta\left(u_{1}\right) .
\end{aligned}
$$

Since $\alpha$ is an algebraic homomorphism and by conditions (HR02), (CC02), we obtain equality (53).

For (HLY1) and (HLY2), by definition we have

$$
\begin{aligned}
{\left[x_{1}+u_{1}, x_{1}+u_{1}\right]=} & {\left[x_{1}, x_{1}\right]+\rho\left(x_{1}\right)\left(u_{1}\right)-\rho\left(x_{1}\right)\left(u_{1}\right)=0, } \\
{\left[x_{1}+u_{1}, x_{1}+u_{1}, x_{3}+u_{3}\right]=} & {\left[x_{1}, x_{1}, x_{3}\right]+D\left(x_{1}, x_{1}\right)\left(u_{3}\right)-\theta\left(x_{1}, x_{3}\right)\left(u_{1}\right) } \\
& +\theta\left(x_{1}, x_{3}\right)\left(u_{1}\right)=0 .
\end{aligned}
$$

For (HLY3), we have

$$
\left[x_{1}+u_{1}, x_{2}+u_{2}, x_{3}+u_{3}\right]_{\omega}+c . p .
$$




$$
\begin{aligned}
= & \left\{\left[x_{1}, x_{2}, x_{3}\right]+\omega\left(x_{1}, x_{2}, x_{3}\right)+D\left(x_{1}, x_{2}\right)\left(u_{3}\right)-\theta\left(x_{1}, x_{3}\right)\left(u_{2}\right)\right. \\
& \left.+\theta\left(x_{2}, x_{3}\right)\left(u_{1}\right)\right\}+c . p .
\end{aligned}
$$

and

$$
\begin{aligned}
& {\left[\left[x_{1}+u_{1}, x_{2}+u_{2}\right]_{\nu}, \alpha\left(x_{3}\right)+\beta\left(u_{3}\right)\right]_{\nu}+c . p . } \\
= & {\left[\left[x_{1}, x_{2}\right]+\rho\left(x_{1}\right)\left(u_{2}\right)-\rho\left(x_{2}\right)\left(u_{1}\right), \alpha\left(x_{3}\right)+\beta\left(u_{3}\right)\right]_{\nu}+c . p . } \\
= & \left\{\left[\left[x_{1}, x_{2}\right], \alpha\left(x_{3}\right)\right]+\nu\left(\left[x_{1}, x_{2}\right], x_{3}\right)+\rho\left(\left[x_{1}, x_{2}\right]\right) \circ \beta\left(u_{3}\right)\right. \\
& \left.-\rho\left(\alpha\left(x_{3}\right)\right) \nu\left(x_{1}, x_{2}\right)-\rho\left(\alpha\left(x_{3}\right)\right) \rho\left(x_{1}\right)\left(u_{2}\right)+\rho\left(\alpha\left(x_{3}\right)\right) \rho\left(x_{2}\right)\left(u_{1}\right)\right\}+c . p .
\end{aligned}
$$

Thus by (HR31) and (CC1) we obtain

$$
\left.\left[x_{1}+u_{1}, x_{2}+u_{2}, x_{3}+u_{3}\right]_{\omega}+\text { c.p. }+\left[x_{1}+u_{1}, x_{2}+u_{2}\right]_{\nu}, \alpha\left(x_{3}\right)+\beta\left(u_{3}\right)\right]_{\nu}+\text { c.p. }=0 .
$$

For (HLY4), we have

$$
\begin{aligned}
& {\left[\left[x_{1}+u_{1}, x_{2}+u_{2}\right]_{\nu}, \alpha\left(x_{3}\right)+\beta\left(u_{3}\right), \alpha\left(y_{1}\right)+\beta\left(v_{1}\right)\right]_{\omega}+c . p . } \\
= & \left\{\left[\left[x_{1}, x_{2}\right], \alpha\left(x_{3}\right), \alpha\left(y_{1}\right)\right]+\omega\left(\left[x_{1}, x_{2}\right], \alpha\left(x_{3}\right), \alpha\left(y_{1}\right)\right)\right. \\
& +D\left(\left[x_{1}, x_{2}\right], \alpha\left(x_{3}\right)\right)\left(\beta\left(v_{1}\right)\right)-\theta\left(\left[x_{1}, x_{2}\right], \alpha\left(y_{1}\right)\right)\left(\beta\left(u_{3}\right)\right) \\
& \left.+\theta\left(\alpha\left(x_{3}\right), \alpha\left(y_{1}\right)\right)\left(\nu\left(x_{1}, x_{2}\right)+\rho\left(x_{1}\right)\left(u_{2}\right)-\rho\left(x_{2}\right)\left(u_{1}\right)\right)\right\}+c . p . \\
= & 0,
\end{aligned}
$$

where the last equality is by (CC2), (HR41) and (HR42).

For (HLY5), we have

$$
\begin{aligned}
& {\left[\alpha\left(x_{1}\right)+\beta\left(u_{1}\right), \alpha\left(x_{2}\right)+\beta\left(u_{2}\right),\left[y_{1}+v_{1}, y_{2}+v_{2}\right]_{\nu}\right]_{\omega} } \\
= & {\left[\alpha\left(x_{1}\right), \alpha\left(x_{2}\right),\left[y_{1}, y_{2}\right]\right]+\underline{\omega\left(\alpha\left(x_{1}\right), \alpha\left(x_{2}\right),\left[y_{1}, y_{2}\right]\right)}+} \\
& \frac{D\left(\alpha\left(x_{1}\right), \alpha\left(x_{2}\right)\right)\left(\nu\left(y_{1}, y_{2}\right)+\rho\left(y_{1}\right)\left(v_{2}\right)-\rho\left(y_{2}\right)\left(v_{1}\right)\right)}{-\theta\left(x_{1},\left[y_{1}, y_{2}\right]\right)\left(u_{2}\right)+\theta\left(x_{2},\left[y_{1}, y_{2}\right]\right)\left(u_{1}\right),}
\end{aligned}
$$

and

$$
\begin{aligned}
& {\left[\left[x_{1}+u_{1}, x_{2}+u_{2}, y_{1}+v_{1}\right]_{\omega}, \alpha^{2}\left(y_{2}\right)+\beta^{2}\left(v_{2}\right)\right]_{\nu} } \\
& +\left[\alpha^{2}\left(y_{1}\right)+\beta^{2}\left(v_{1}\right),\left[x_{1}+u_{1}, x_{2}+u_{2}, y_{2}+v_{2}\right]_{\omega}\right]_{\nu} \\
= & {\left[\left[x_{1}, x_{2}, y_{1}\right], \alpha^{2}\left(y_{2}\right)\right]+\underline{\nu\left(\left[x_{1}, x_{2}, y_{1}\right], \alpha^{2}\left(y_{2}\right)\right)}+\rho\left(\left[x_{1}, x_{2}, y_{1}\right]\right)\left(v_{2}\right) } \\
& \frac{-\rho\left(y_{2}\right)\left(\omega\left(x_{1}, x_{2}, y_{1}\right)+D\left(x_{1}, x_{2}\right)\left(v_{1}\right)-\theta\left(x_{1}, y_{1}\right)\left(u_{2}\right)+\theta\left(x_{2}, y_{1}\right)\left(u_{1}\right)\right)}{+\left[\alpha^{2}\left(y_{1}\right),\left[x_{1}, x_{2}, y_{2}\right]\right]}+\underline{\nu\left(\alpha^{2}\left(y_{1}\right),\left[x_{1}, x_{2}, y_{2}\right]\right)} \\
& +\rho\left(y_{1}\right)\left(\omega\left(x_{1}, x_{2}, y_{2}\right)\right. \\
& -\rho\left(\left[x_{1}, x_{2}, y_{2}\right]\right)\left(v_{1}\right) .
\end{aligned}
$$

Thus by (CC3), (HR51) and (HR52) we obtain

$$
\begin{aligned}
& {\left[\alpha\left(x_{1}\right)+\beta\left(u_{1}\right), \alpha\left(x_{2}\right)+\beta\left(u_{2}\right),\left[y_{1}+v_{1}, y_{2}+v_{2}\right]_{\nu}\right]_{\omega} } \\
= & {\left[\left[x_{1}+u_{1}, x_{2}+u_{2}, y_{1}+v_{1}\right]_{\omega}, \alpha^{2}\left(y_{2}\right)+\beta^{2}\left(v_{2}\right)\right]_{\nu} } \\
& +\left[\alpha^{2}\left(y_{1}\right)+\beta^{2}\left(v_{1}\right),\left[x_{1}+u_{1}, x_{2}+u_{2}, y_{2}+v_{2}\right]_{\omega}\right]_{\nu}
\end{aligned}
$$

Therefore (HLY5) is valid. 
Now it suffices to verify (HLY6). By definition,

$$
\begin{aligned}
& {\left[\alpha^{2}\left(x_{1}\right)+\beta^{2}\left(u_{1}\right), \alpha^{2}\left(x_{2}\right)+\beta^{2}\left(u_{2}\right),\left[y_{1}+v_{1}, y_{2}+v_{2}, y_{3}+v_{3}\right]\right]} \\
& =\left[\alpha^{2}\left(x_{1}\right), \alpha^{2}\left(x_{2}\right),\left[y_{1}, y_{2}, y_{3}\right]\right]+\underline{\omega\left(\alpha^{2}\left(x_{1}\right), \alpha^{2}\left(x_{2}\right),\left[y_{1}, y_{2}, y_{3}\right]\right)} \\
& -\theta\left(\alpha^{2}\left(x_{1}\right),\left[y_{1}, y_{2}, y_{3}\right]\right)\left(\beta^{2}\left(u_{2}\right)\right)+\theta\left(\alpha^{2}\left(x_{2}\right),\left[y_{1}, y_{2}, y_{3}\right]\right)\left(\beta^{2}\left(u_{1}\right)\right) \\
& \left.+\underline{+D\left(\alpha^{2}\left(x_{1}\right), \alpha^{2}\left(x_{2}\right)\right)\left(\omega\left(y_{1}, y_{2}, y_{3}\right)\right.}+D\left(y_{1}, y_{2}\right)\left(v_{3}\right)-\theta\left(y_{1}, y_{3}\right)\left(v_{2}\right)+\theta\left(y_{2}, y_{3}\right)\left(v_{1}\right)\right) \text {, } \\
& {\left[\left[x_{1}+u_{1}, x_{2}+u_{2}, y_{1}+v_{1}\right], \alpha^{2}\left(y_{2}\right)+\beta^{2}\left(v_{2}\right), \alpha^{2}\left(y_{3}\right)+\beta^{2}\left(v_{3}\right)\right]} \\
& \left.\left.=\left[\left[x_{1}, x_{2}, y_{1}\right], \alpha^{2}\left(y_{2}\right), \alpha^{2}\left(y_{3}\right)\right]\right]+\underline{\omega}\left(\left[x_{1}, x_{2}, y_{1}\right], \alpha^{2}\left(y_{2}\right), \alpha^{2}\left(y_{3}\right)\right]\right) \\
& -D\left(\left[x_{1}, x_{2}, y_{1}\right], \alpha^{2}\left(y_{2}\right)\right)\left(\beta^{2}\left(v_{3}\right)\right)+\theta\left(\left[x_{1}, x_{2}, y_{1}\right], \alpha^{2}\left(y_{3}\right)\right)\left(\beta^{2}\left(u_{1}\right)\right) \\
& +\theta\left(\alpha^{2}\left(y_{2}\right), \alpha^{2}\left(y_{3}\right)\right)\left(\omega\left(x_{1}, x_{2}, y_{1}\right)+D\left(x_{1}, x_{2}\right)\left(v_{1}\right)-\theta\left(x_{1}, y_{1}\right)\left(u_{2}\right)+\theta\left(x_{2}, y_{1}\right)\left(u_{1}\right)\right) \text {, } \\
& {\left[\alpha^{2}\left(y_{1}\right)+\beta^{2}\left(v_{1}\right),\left[x_{1}+u_{1}, x_{2}+u_{2}, y_{2}+v_{2}\right], \alpha^{2}\left(y_{3}\right)+\beta^{2}\left(v_{3}\right)\right]} \\
& =\left[\alpha^{2}\left(y_{1}\right),\left[x_{1}, x_{2}, y_{2}\right], \alpha^{2}\left(y_{3}\right)\right]+\omega\left(\alpha^{2}\left(y_{1}\right),\left[x_{1}, x_{2}, y_{2}\right], \alpha^{2}\left(y_{3}\right)\right) \\
& +D\left(\alpha^{2}\left(y_{1}\right),\left[x_{1}, x_{2}, y_{2}\right]\right)\left(\beta^{2}\left(v_{3}\right)\right)+\theta\left(\left[x_{1}, x_{2}, y_{2}\right], \alpha^{2}\left(y_{3}\right)\right)\left(\beta^{2}\left(v_{1}\right)\right) \\
& -\theta\left(\alpha^{2}\left(y_{1}\right), \alpha^{2}\left(y_{3}\right)\right)\left(\omega\left(x_{1}, x_{2}, y_{2}\right)+D\left(x_{1}, x_{2}\right)\left(v_{2}\right)-\theta\left(x_{1}, y_{2}\right)\left(u_{2}\right)+\theta\left(x_{2}, y_{2}\right)\left(u_{1}\right)\right) \text {, } \\
& {\left[\alpha^{2}\left(y_{1}\right)+\beta^{2}\left(v_{1}\right), \alpha^{2}\left(y_{2}\right)+\beta^{2}\left(v_{2}\right),\left[x_{1}+u_{1}, x_{2}+u_{2}, \alpha^{2}\left(y_{3}\right)+\beta^{2}\left(v_{3}\right)\right]\right]} \\
& =\left[\alpha^{2}\left(y_{1}\right)+\alpha^{2}\left(y_{2}\right),\left[x_{1}, x_{2}, y_{3}\right]\right]+\underline{\omega\left(\alpha^{2}\left(y_{1}\right)+\alpha^{2}\left(y_{2}\right),\left[x_{1}, x_{2}, y_{3}\right]\right)} \\
& -\theta\left(\alpha^{2}\left(y_{1}\right),\left[x_{1}, x_{2}, y_{3}\right]\right)\left(\beta^{2}\left(v_{2}\right)\right)+\theta\left(\alpha^{2}\left(y_{2}\right),\left[x_{1}, x_{2}, y_{3}\right]\right)\left(\beta^{2}\left(v_{1}\right)\right) \\
& +D\left(\alpha^{2}\left(y_{1}\right), \alpha^{2}\left(y_{2}\right)\right)\left(\omega\left(x_{1}, x_{2}, y_{3}\right)+D\left(x_{1}, x_{2}\right)\left(v_{3}\right)-\theta\left(x_{1}, y_{3}\right)\left(u_{2}\right)+\theta\left(x_{2}, y_{3}\right)\left(u_{1}\right)\right),
\end{aligned}
$$

It follows that

$$
\begin{aligned}
& {\left[\alpha^{2}\left(x_{1}\right)+\beta^{2}\left(u_{1}\right), \alpha^{2}\left(x_{2}\right)+\beta^{2}\left(u_{2}\right),\left[y_{1}+v_{1}, y_{2}+v_{2}, y_{3}+v_{3}\right]\right] } \\
= & {\left[\left[x_{1}+u_{1}, x_{2}+u_{2}, y_{1}+v_{1}\right], \alpha^{2}\left(y_{2}\right)+\beta^{2}\left(v_{2}\right), \alpha^{2}\left(y_{3}\right)+\beta^{2}\left(v_{3}\right)\right] } \\
& +\left[\alpha^{2}\left(y_{1}\right)+\beta^{2}\left(v_{1}\right),\left[x_{1}+u_{1}, x_{2}+u_{2}, y_{2}+v_{2}\right], y_{3}+v_{3}\right] \\
& +\left[\alpha^{2}\left(y_{1}\right)+\beta^{2}\left(v_{1}\right), \alpha^{2}\left(y_{2}\right)+\beta^{2}\left(v_{2}\right),\left[x_{1}+u_{1}, x_{2}+u_{2}, \alpha^{2}\left(y_{3}\right)+\beta^{2}\left(v_{3}\right)\right]\right]
\end{aligned}
$$

by (CC4), (HR61) and (HR62). Therefore we obtain a HLYA on $T \oplus V$ under the maps (49), (50) and (51). The proof is completed.

Lemma 4.6. Two abelian extensions of $H L Y A s 0 \rightarrow V \rightarrow T \oplus_{(\nu, \omega)} V \rightarrow T \rightarrow 0$ and $0 \rightarrow V \rightarrow T \oplus_{\left(\nu^{\prime}, \omega^{\prime}\right)}$ $V \rightarrow T \rightarrow 0$ are equivalent if and only if $(\nu, \omega)$ and $\left(\nu^{\prime}, \omega^{\prime}\right)$ are in the same cohomology class.

Proof. Assume the two extensions are equivalent, we choose $F: T \oplus_{(\nu, \omega)} V \rightarrow T \oplus_{\left(\nu^{\prime}, \omega^{\prime}\right)} V$ to be the corresponding homomorphism. Then we get

$$
\begin{aligned}
& F\left[x_{1}, x_{2}\right]_{\nu}=\left[F\left(x_{1}\right), F\left(x_{2}\right)\right]_{\nu^{\prime}}, \\
& F\left[x_{1}, x_{2}, x_{3}\right]_{\omega}=\left[F\left(x_{1}\right), F\left(x_{2}\right), F\left(x_{3}\right)\right]_{\omega^{\prime}} .
\end{aligned}
$$

Since $F$ is an equivalence of extensions, there exist $f: T \rightarrow V$ such that

$$
F\left(x_{i}+u\right)=x_{i}+f\left(x_{i}\right)+u, \quad \forall x_{i} \in T
$$


Now the equation (55) is equal to

$$
\begin{aligned}
& {\left[x_{1}, x_{2}\right]+f\left(\left[x_{1}, x_{2}\right]\right)+\nu\left(x_{1}, x_{2}\right) } \\
= & {\left[x_{1}, x_{2}\right]+\nu^{\prime}\left(x_{1}, x_{2}\right)+\rho\left(x_{1}\right) f\left(x_{2}\right)-\rho\left(x_{2}\right) f\left(x_{1}\right) . }
\end{aligned}
$$

Thus we have

$$
\left(\nu-\nu^{\prime}\right)\left(x_{1}, x_{2}\right)=\rho\left(x_{1}\right) f\left(x_{2}\right)-\rho\left(x_{2}\right) f\left(x_{1}\right)-f\left(\left[x_{1}, x_{2}\right]\right) .
$$

The equation (56) is equalent to

$$
\begin{aligned}
& {\left[x_{1}, x_{2}, x_{3}\right]+\omega\left(x_{1}, x_{2}, x_{3}\right)+f\left(\left[x_{1}, x_{2}, x_{3}\right]\right) } \\
= & {\left[x_{1}, x_{2}, x_{3}\right]+\omega^{\prime}\left(x_{1}, x_{2}, x_{3}\right) } \\
& +D\left(x_{1}, x_{2}\right) f\left(x_{3}\right)-\theta\left(x_{1}, x_{3}\right) f\left(x_{2}\right)+\theta\left(x_{2}, x_{3}\right) f\left(x_{1}\right) .
\end{aligned}
$$

Thus we have

$$
\begin{aligned}
& \left(\omega-\omega^{\prime}\right)\left(x_{1}, x_{2}, x_{3}\right) \\
= & D\left(x_{1}, x_{2}\right) f\left(x_{3}\right)-\theta\left(x_{1}, x_{3}\right) f\left(x_{2}\right)+\theta\left(x_{2}, x_{3}\right) f\left(x_{1}\right)-f\left(\left[x_{1}, x_{2}, x_{3}\right]\right) .
\end{aligned}
$$

Therefore $(\nu, \omega)$ and $\left(\nu^{\prime}, \omega^{\prime}\right)$ are in the same cohomology class. Conversely, if $(\nu, \omega)$ and $\left(\nu^{\prime}, \omega^{\prime}\right)$ are in the same cohomology class, then we can show that $F$ is an equivalence. We omit the details.

Finally, we obtain the main result of this section:

Theorem 4.7. Let $T$ be a HLYA and $V$ a T-module. Then there is a one-to-one correspondence between the set of equivalence classes of abelian extensions of the HLYA and the (2,3)-cohomology group. More precisely, there is a bijection map

$$
\operatorname{Ext}(T, V) \rightarrow H^{2}(T, V) \times H^{3}(T, V) .
$$

Therefore, the abelian extensions of $T$ by $V$ are classified by the $(2,3)$-cohomology group.

\section{Acknowledgements}

The research was supported by Doctoral Research Program (5101019170129) of Henan Normal University.

\section{References}

[1] H. Ataguema, A. Makhlouf, and S. Silvestrov, Generalization of $n$-ary Nambu algebras and beyond, J. Math. Phys. 50(2009), 083501.

[2] J. Arnlind, A. Makhlouf, and S. Silvestrov, Ternary Hom-Nambu-Lie algebras induced by Hom-Lie algebras, J. Math. Phys. 51(2010), 043515.

[3] F. Ammar, S. Mabrouk, A. Makhlouf, Representations and cohomology of $n$-ary multiplicative Hom-Nambu-Lie algebras, J. Geom. Physics, 61(2011), 1898-1913.

[4] P. Benito, A. Elduque, and F. Martín-Herce, Irreducible Lie-Yamaguti algebras, J. Pure Appl. Algebra 213(2009), 795-808.

[5] S. Caenepeel and I. Goyvaerts, Monoidal Hom-Hopf Algebras, Commu. Algebra 39(2011): $2216-2240$. 
[6] I. Dorfman, Dirac Structures and Integrability of Nonlinear Evolution Equation. John Wiley \& Sons, Ltd., Chichester, 1993.

[7] M. Gerstenhaber, On the deformation of rings and algebras, Ann. Math. 79(1964): 59-103.

[8] D. Gaparayi and A. Nourou Issa, A twisted Generalization of Lie-Yamaguti algebras, Int. J. Algebra, 6(2012), 339-352.

[9] J. T. Hartwig, D. Larsson, S. D. Silvestrov, Deformations of Lie algebras using $\sigma$-derivations, J. Algebra 295(2006), 314-361.

[10] M. Kikkawa, Geometry of homogeneous Lie loops, Hiroshima Math. J. 5(1975), no. 2, $141-179$.

[11] M.K. Kinyon and A. Weinstein, Leibniz algebras, Courant algebroids, and multiplications on reductive homogeneous spaces, Amer. J. Math. 123(2001), no. 3, 525-550.

[12] Y. Ma, L.Y. Chen and J. Lin, One-parameter formal deformations of Hom-Lie-Yamaguti algebras, J. Math. Phys. 56(2015), 011701.

[13] A. Nijenhuis and R. W. Richardson, Cohomology and deformations in graded Lie algebras, Bull. Amer. Math. Soc. 72(1966), 1-29.

[14] K. Nomizu, Invariant affine connections on homogeneous spaces, Amer. J. Math 76(1954), 33-65.

[15] Y. Sheng, Representations of hom-Lie algebras, Algebra and Representation Theory, 15(6)(2012), 1081-1098.

[16] K. Yamaguti, On the Lie triple system and its generalization, J. Sci. of Hiroshima Univ., Ser. A, v. 21, 1958, pp. 155-160.

[17] K. Yamaguti, On cohomology groups of general Lie triple systems, Kumamoto J. Sci., A 8(1969), 135-146.

[18] D. Yau, Hom-algebras and homology, J. Lie Theory 19(2009), 409-421.

[19] D. Yau, On n-ary Hom-Nambu and Hom-Nambu-Lie algebras, J. Geom. Phys. 62(2012), 506522.

[20] T. Zhang, Notes on Cohomologies of Lie Triple Systems, J. Lie Theory, 24(4)(2014), 909-929.

[21] T. Zhang and J. Li, Deformations and extensions of Lie-Yamaguti algebras, to appear in Linear and Multilinear Algebra. DOI:10.1080/03081087.2014.1000815 